\title{
tic\&société
}

Vol. 14, $\mathbf{N}^{\circ}$ 1-2 | 1er semestre 2020 - 2ème semestre 2020

Mutations numériques de la musique : des

contradictions à analyser

\section{Variations autour de l'adoption de plateformes de vente en ligne : la réception d'un nouveau dispositif marchand par les entrepreneurs albigeois}

Reactions of Albi entrepreneurs to a new online sales platform

Variaciones en torno a la adopción de plataformas de venta online: la recepción de un nuevo dispositivo comercial por parte de los empresarios albigenses

Cédric CALVIGNAC et Mathieu VIDAL

\section{OpenEdition}

Journals

Édition électronique

URL : http://journals.openedition.org/ticetsociete/5271

DOI : 10.4000/ticetsociete.5271

Éditeur

Association ARTIC

Édition imprimée

Pagination : 303-342

Référence électronique

Cédric CALVIGNAC et Mathieu VIDAL, «Variations autour de l'adoption de plateformes de vente en ligne : la réception d'un nouveau dispositif marchand par les entrepreneurs albigeois », tic\&société [En ligne], Vol. 14, N 1-2 I 1er semestre 2020 - 2ème semestre 2020, mis en ligne le 11 novembre 2020, consulté le 24 février 2021. URL : http://journals.openedition.org/ticetsociete/5271 ; DOI : https:// doi.org/10.4000/ticetsociete.5271 
tic\&société - 14(1-2), 2020

\title{
Variations autour de l'adoption de plateformes de vente en ligne : la réception d'un nouveau dispositif marchand par les entrepreneurs albigeois
}

\begin{abstract}
Cédric CALVIGNAC
Cédric Calvignac est maître de conférences en sociologie à I'Institut national universitaire Jean-François Champollion d'Albi (Université fédérale Toulouse Midi-Pyrénées). Il est membre du Centre d'étude et de Recherche Travail, Organisation, Pouvoir (CERTOP - UMR 5044) et du groupe de recherche pluridisciplinaire PPES. Ses travaux s'inscrivent à la croisée de la sociologie des sciences et des techniques (STS), de la sociologie de l'innovation et de la sociologie économique. Ils portent sur la conception, l'usage et la maintenance de différentes technologies (notamment des TIC), sur les innovations faites par et pour les usagers (réseaux wifi citoyens, logiciel libre), sur l'adoption d'outils numériques par des publics variés (plateformes électroniques de vente, applications touristiques mobiles, dispositifs d'automesure de soi) et, plus généralement, sur la place qu'occupe la technique dans le cours ordinaire des pratiques sociales. cedric.calvignac@univ-tlse2.fr
\end{abstract}

\section{Mathieu VIDAL}

Mathieu Vidal est maître de conférences en géographie et aménagement à I'Institut national universitaire Jean-François Champollion d'Albi. II est membre du LISST-CIEU (UMR 5193) et du groupe de recherche pluridisciplinaire PPES. II est par ailleurs président de l'association Villes Internet, qui œuvre à la sensibilisation des collectivités territoriales aux enjeux du numérique et à la valorisation de leurs actions (via le label Villes Internet). Ses travaux portent notamment sur les usages et les usagers du numérique ainsi que sur le déploiement des politiques publiques et des services (aux) publics, en particulier dans le contexte de la ville de demain et des territoires connectés. mathieu.vidal@univ-jfc.fr 
Variations autour de l'adoption de plateformes de vente en ligne : la réception d'un nouveau dispositif marchand par les entrepreneurs albigeois

\title{
Variations autour de l'adoption de plateformes de vente en ligne : la réception d'un nouveau dispositif marchand par les entrepreneurs albigeois
}

Résumé : Les plateformes de vente en ligne (ou marketplaces) figurent aujourd'hui parmi les dispositifs de captation marchande les plus performants de l'économie numérique. Dans différents secteurs d'activités (tourisme, transports, biens culturels), elles se sont très largement imposées pour parfois devenir dominantes. Le présent article cherche à analyser les déterminants de leur adoption par les responsables d'entreprises de l'agglomération albigeoise. Partant d'une enquête quantitative, l'article examine trois grands types de variations : 1) la variation des facteurs individuels, organisationnels et structurels qui président à l'adoption du dispositif ; 2) la variation de l'intérêt manifesté par les entrepreneurs pour deux types de plateformes distinctes: les marketplaces multi-sites (de type Amazon) et une marketplace locale pensée pour relocaliser l'activité économique sur le territoire albigeois ; 3) la variation inhérente à l'analyse comparée de données factuelles (adoption effective des marketplaces multi-sites) et prospectives (déclaration d'intérêt pour un projet de marketplace locale).

Mots-clés : e-commerce, plateforme, adoption, vente, agencement marchand.

\section{Reactions of Albi entrepreneurs to a new online sales platform}

\begin{abstract}
Online sales platforms (or marketplaces) are among the most efficient market devices in today's digital economy. In
\end{abstract}


different sectors of activity (tourism, transport, culture), they have become widely used and in some cases dominant. This article seeks to analyse the determinants of their adoption by different entrepreneurs in the agglomeration of Albi. Based on a quantitative survey, the article examines three main types of variations: 1) the variation in the individual, organizational and structural factors that determine the adoption of online platforms; 2) the variation in entrepreneurs' interest in two distinct types of platforms: multi-sited marketplaces (such as Amazon) and a local marketplace designed to relocate economic activity; 3 ) the variation related to the comparative analysis of factual data (effective adoption of multi-sited marketplaces) and prospective data (statement of interest for a local marketplace project).

Keywords: e-commerce, platform, adoption, retail, market agencement.

\section{Variaciones en torno a la adopción de plataformas de venta online: la recepción de un nuevo dispositivo comercial por parte de los empresarios albigenses}

Resumen: Las plataformas de venta en línea (o mercados) se encuentran entre los dispositivos de comercio más eficientes de la economía digital. Las plataformas se han establecido en diferentes sectores de actividad (turismo, transporte, bienes culturales), llegando incluso a ser dominantes. Este artículo analiza los factores determinantes de su adopción por parte de los empresarios de la aglomeración albigense. Basado en una encuesta cuantitativa, el artículo examina tres tipos principales de variaciones: 1) la variación en los factores individuales, organizacionales y estructurales que se sitúan en la base de la adopción del dispositivo; 2) la variación en el interés mostrado por los empresarios en dos tipos distintos de plataformas: las plataformas multi-sitio (Amazon) y las plataformas locales, concebidas para relocalizar la actividad económica; 3) la variación inherente al análisis comparativo de los datos fácticos 
Variations autour de l'adoption de plateformes de vente en ligne : la réception d'un nouveau dispositif marchand par les entrepreneurs albigeois

(la adopción real de los mercados multi-sitio) y de los datos prospectivos (la declaración del interés por un proyecto local).

Palabras claves: comercio electrónico, plataforma, adopción, venta, objetivo comercial. 
Au cours des dernières années, le poids du e-commerce dans l'économie française s'est notablement accru. Selon la FEVAD ${ }^{1}$, la part que représente le e-commerce sur l'ensemble des ventes au détail est en effet passée de $6 \%$ en 2014 à 8,5 \% en 2017. Cette évolution à la hausse résulte d'une forte augmentation du nombre de sites marchands actifs ${ }^{2}$, mais aussi, et surtout, de la croissance remarquable d'un très petit nombre d'entre eux, qui engendre un véritable effet de concentration du marché ${ }^{3}$. Dans sa forme la plus avancée, un tel effet de concentration procède bien souvent de l'émergence et de la domination de plateformes de vente en ligne (ou marketplaces ${ }^{4}$ ) qui agrègent en leur sein un large ensemble d'offres et parviennent ainsi à capter une foule considérable de visiteurs et d'acheteurs. En France, les plateformes de vente en ligne ont enregistré pas moins de $28 \%$ du volume d'affaires total réalisé par les sites de e-commerce en 2017, soit une progression de 15 points par rapport à 2015 (FEVAD, 2017). Ces nouveaux intermédiaires marchands sont donc devenus, en très peu de temps, des points de fixation stratégiques au cœur du vaste réseau de l'économie numérique (Benavent, 2016; Evans et Schmalensee, 2016; Parker, Van Alstyne et Choudary, 2016 ; Srnicek, 2017). À tel point que de nombreux observateurs s'alarment aujourd'hui des possibles dérives monopolistiques de ces plateformes, soulignent les dangers inhérents à la constitution par celles-ci de positions dominantes sur le marché (Boullier, 2016 ; Maxwell et Pénard, 2016).

Les plateformes de vente en ligne se sont le plus souvent imposées dans des secteurs d'activités déjà fortement convertis au e-commerce. C'est notamment le cas des secteurs du

\footnotetext{
${ }^{1}$ Fédération du e-commerce et de la vente à distance.

2 On passe de 157000 sites en 2014 à 204000 en 2016.

3 En 2017, 0,6\% des sites marchands captaient $65,2 \%$ du chiffre d'affaires ecommerce en France (FEVAD, 2018).

${ }^{4} \mathrm{Ici}$, nous reprenons à notre compte le terme marketplace - très largement cité dans la littérature grise et dans les médias - alors même que son emploi est fautif puisqu'il désigne en anglais, au-delà des seules plateformes de vente en ligne (emarketplaces), l'ensemble des places de marché physiques et numériques (marketplaces). Nous conserverons malgré tout ce terme, car, pour impropre que soit cette traduction, elle s'est finalement imposée en France au point de désigner de façon univoque et exclusive les seules places de marché numériques.
} 
Variations autour de l'adoption de plateformes de vente en ligne : la réception d'un nouveau dispositif marchand par les entrepreneurs albigeois

tourisme, des transports, des produits culturels ${ }^{5}$, des textiles de maison, des équipements high-tech ou encore de l'habillement. Dans ces différents secteurs, la part du chiffre d'affaires imputable au e-commerce est particulièrement importante (allant de 16 à $44 \%^{6}$ ) et les achats en ligne se réalisent fréquemment sur des plateformes de vente dédiées (Booking, Airbnb, Uber, Amazon, Fnac). Ce franc succès des plateformes de vente en ligne a occasionné, dans les domaines précités, un renouvellement conséquent du paysage de l'offre: une part importante des marketplaces ont en effet été créées par une nouvelle génération d'entreprises qui présentent la particularité de n'avoir jamais été présentes que sur Internet (pure players ${ }^{7}$ ). Le développement de ces plateformes a également contribué à instaurer de nouvelles conditions d'échange, à modifier le comportement des consommateurs (Méadel, 2006). De nos jours, pour nombre de leurs achats, les consommateurs vont et viennent entre différents sites de e-commerce et différents magasins implantés sur le territoire (click \& mortar) : ils observent une démarche de consommation multicanal propre à mieux équiper leurs choix (Van Heems, 2012). Ajoutons également que les consommateurs font aujourd'hui davantage part de leur appréciation des biens et des services achetés par l'intermédiaire de systèmes de notation et de commentaires devenus en très peu de temps - et sous l'impulsion de ces mêmes plateformes - un véritable allant de soi du e-commerce (Beauvisage et al., 2013, Beuscart, Dagiral et Parasie, 2016 ; Luca, 2017). Enfin, différentes marketplaces ont su encadrer et favoriser les échanges entre particuliers et ont directement contribué à l'émergence d'une offre portée par une foule d'amateurs ou par des " professionnels-amateurs » s'essayant à des formes d'entrepreneuriat naissant (Calvignac, 2012a, 2012b ; Flichy, 2010 ; Leadbeater et Miller, 2004).

\footnotetext{
${ }^{5}$ Voir le numéro de tic\&société sur la « plateformisation » des industries culturelles (Bullich et Schmitt, 2019) ainsi que les deux numéros que la revue Réseaux consacre aux différentes " activités menées sur les plateformes numériques » (Beuscart et Flichy, 2018).

${ }^{6}$ Le rapport de la FEVAD, portant sur l'année 2016, estime la part du e-commerce à $44 \%$ dans le secteur du tourisme, à $43 \%$ dans le secteur des produits culturels, à $27 \%$ dans le secteur des textiles de maison, à $22 \%$ dans le secteur des produits de maison high tech, à $17 \%$ dans le secteur de l'électroménager, à $16 \%$ dans le secteur de l'habillement et à seulement $5 \%$ dans celui des produits de grande consommation (FEVAD, 2017).

7 L'Insee indique que, dès 2012, les pure players « dominent les ventes web du commerce de détail » (Insee, 2014).
} 
Plus qu'une simple déclinaison numérique du centre commercial ou du supermarché, la marketplace est un véritable " objet-frontière " facteur de réagencements marchands inédits (Star et Griesemer, 1989). Se pencher sur l'adoption de ce nouvel outil de vente revient donc à saisir à nouveau frais le processus de "coprofilage" des acteurs engagés dans l'animation des marchés bifaces (Caillaud et Jullien, 2003; Callon, 2017 ; Rochet et Tirole, 2003 ; Schmalensee et Evans, 2007). Dans le cadre de cet article, nous concentrerons notre attention sur l'une des faces de ces marchés : celle relevant de la relation entre les administrateurs des plateformes et les entreprises clientes (B2B). Nous resterons donc du côté des vendeurs pour identifier les déterminants présidant à leur adoption (ou non) des marketplaces.

Notre contribution s'inscrit dans la lignée des travaux qui ont récemment montré que, du côté de l'offre, la participation à ces plateformes de vente en ligne répondait à des degrés d'engagement extrêmement variables (Boullier, 2016 ; Cardon et Casilli, 2015 ; Flichy, 2017). En effet, quand certains participants ne vendent qu'une poignée de leurs articles en ligne, d'autres déclinent sur Internet toute l'étendue de leur offre en magasin (voire l'augmentent de quelques articles uniquement disponibles sur Internet). Quand certains font de ces plateformes un moyen d'asseoir une réputation déjà acquise, d'autres en font de formidables tremplins pour le lancement de leur entreprise ou la conversion professionnelle de leur activité semi-amatrice (Flichy, 2017). On le voit, l'adoption de ces plateformes est à mettre directement en rapport avec l'identité, le positionnement et les objectifs des entrepreneurs concernés. Le statut des responsables d'entreprise, leur plan de développement commercial, leur désignation des publics cibles, leurs habiletés techniciennes ou encore leur sentiment à l'égard du e-commerce peuvent en effet peser sur l'emploi (ou non) d'une marketplace. Plus largement, et cette fois au niveau de l'entreprise, des questions de taille, d'implantation territoriale, d'appartenance sectorielle ou encore de stratégies commerciales peuvent expliquer la grande variabilité des taux d'adoption de ces nouveaux dispositifs marchands. À notre connaissance, aucune enquête n'a encore interrogé l'ensemble des déterminants individuels (identité et compétences du responsable), organisationnels (situation de l'entreprise, modes de production, stratégies commerciales) et structurels (appartenance 
Variations autour de l'adoption de plateformes de vente en ligne : la réception d'un nouveau dispositif marchand par les entrepreneurs albigeois

sectorielle, degré de concurrence) qui interviennent dans la décision de faire siennes ces nouvelles interfaces commerciales. C'est ce que nous nous proposons de faire au travers de l'analyse d'une enquête par questionnaire réalisée en 2017 auprès de 193 responsables d'entreprises situées dans la communauté d'agglomération du Grand Albigeois.

L'originalité de notre enquête tient au fait qu'elle permet d'examiner trois types de variations. Un premier ensemble de variations porte, comme précisé plus haut, sur les multiples facteurs qui président à l'adoption de l'outil plateforme comme instrument de vente. Un deuxième type de variations s'exerce également du côté du design des plateformes, de leurs qualités et de leurs limites constitutives. Pour illustrer ce point, nous rendrons compte de l'intérêt manifesté par les entrepreneurs pour deux types de plateformes distinctes: d'un côté, des solutions de marketplaces multi-sites ${ }^{8}$ très largement diffusées (Amazon, eBay, Booking, etc.) ; de l'autre, une solution de marketplace locale qui, encore à l'état de projet, serait chargée d'offrir une plus grande visibilité et un meilleur accès aux produits et aux services locaux et, par là même, de relocaliser une part des activités économiques sur le territoire albigeois. Nous verrons que l'attrait pour ces deux types de plateformes diffère tout autant que le profil des entrepreneurs y adhérant. Enfin, le troisième ensemble de variations étudié a trait à la nature des réponses examinées, car si l'intérêt pour les marketplaces multisites peut se mesurer à l'aune de l'adhésion effective à des solutions existantes (usage réel), l'intérêt pour une marketplace locale reste encore, pour ce qui est du marché albigeois, cantonné à la déclaration d'intérêt pour une solution à venir, pour un projet papier encore largement indéfini (perspective d'usage).

\footnotetext{
${ }^{8}$ La différence entre les deux types de marketplaces identifiés a principalement trait aux objectifs poursuivis par les porteurs de projet. Dans le cas des plateformes multisites domine une logique commerciale prenant la forme d'une dynamique d'expansion " globale » (mettre en place ici et là ce que l'on a élaboré ailleurs), ou "glocale » (s'appuyer sur un modèle générique et le modifier à la marge en fonction des particularités des lieux d'implantation). A contrario, dans le cas du projet de plateforme locale étudié, il est question d'une logique de capitalisation et de valorisation des ressources (y compris humaines) d'un territoire unique. Autrement dit, si les plateformes multi-sites se préoccupent généralement assez peu du développement économique des territoires investis (seuls les coûts logistiques associés aux transactions participent de l'attention portée à la dimension spatiale de l'échange), ce n'est bien évidemment pas le cas d'une plateforme locale pensée pour redynamiser l'activité économique d'un lieu singulier selon des modalités de distribution plus responsables et durables (circuits courts).
} 
Nous aurons donc la possibilité d'analyser ce qui sépare deux types de données: des données factuelles (adoption effective des marketplaces multi-sites) et des données prospectives (chances d'adoption d'une marketplace locale en gestation). La considération de ces trois variations autour de l'adoption des plateformes de vente en ligne nous permettra de mieux comprendre comment ce dispositif singulier entre ou non en résonance avec les différentes trajectoires des entreprises étudiées.

\section{Méthodologie}

Notre enquête par questionnaire s'est déroulée au cours des mois de janvier à mars 2017. Elle visait à identifier les techniques de vente et les stratégies commerciales employées par les entrepreneurs du Grand Albigeois avec une attention toute particulière accordée aux mesures effectivement prises en matière d' " électronisation du commerce » (Hagberg, Sundstrom et Egels-Zandén, 2016; Rallet, 2001). Le questionnaire comportait quatre grandes parties : la première était destinée à décrire l'entreprise et son marché (taille, secteur d'activités, origine des produits, valorisation des ressources locales); la deuxième portait sur l'inscription des entrepreneurs dans différents collectifs ou démarches corporatives (adhésion à une association de commerçants, à une chambre consulaire, à un réseau de franchise); la troisième s'intéressait aux différents dispositifs techniques mobilisés (vitrine, publicité, site Internet, plateforme, label); la quatrième rendait compte de l'intérêt manifesté par les entrepreneurs pour un projet de marketplace locale porté par la communauté d'agglomération du Grand Albigeois. C'est à partir du croisement de ces quatre sousensembles de données que nous avons pu identifier au sein de la population des entrepreneurs albigeois des écarts d'appréciation notables à l'égard des deux types de marketplaces distingués.

L'essentiel de nos développements repose sur l'analyse de deux variables à expliquer : 1) l'usage (ou non) de marketplaces multi-sites ; 2) la déclaration d'intérêt (ou non) pour un projet de marketplace locale. Avant d'analyser les réponses accordées à ces deux questions, il nous faut produire un travail d'explicitation des termes et des expressions choisis. Ce n'est qu'à cette 
Variations autour de l'adoption de plateformes de vente en ligne : la réception d'un nouveau dispositif marchand par les entrepreneurs albigeois

condition que le lecteur pourra appréhender notre travail en toute connaissance de cause.

\subsection{De l'incidence du vocabulaire choisi}

$\mathrm{Si}$, dans le cadre de cet article, nous employons indifféremment les expressions «plateformes de vente en ligne " et "marketplaces", il en a été autrement au cours de l'enquête. Nous avons, au moment du recueil des données, et pour faciliter la compréhension des entrepreneurs interrogés, recouru exclusivement à l'expression française. Or l'emploi du terme plateforme - même s'il demeure à nos yeux le plus pertinent des choix - n'est pas sans susciter quelques interrogations et inquiétudes quant à l'univocité de son interprétation par les répondants. Les répondants s'accordent-ils sur une définition commune de ce qu'est une plateforme de vente en ligne? Envisagent-ils tous de la même manière les outils figurant sous cette appellation ? On peut en douter. En effet, et à la suite de Tarleton Gillespie (2010), rappelons que le terme plateforme brille tant par son pouvoir d'évocation (ce qu'il permet de figurer) que par son caractère indéfini (on ne sait pas exactement ce qu'il recouvre $)^{9}$. Ce terme est donc susceptible de générer des interprétations différenciées, voire concurrentes.

Au moment de la conception de notre questionnaire, nous avons malgré tout choisi de nous conformer au vocabulaire indigène le plus couramment employé (plateforme). Cela étant, et pour éviter de trop grands écarts d'interprétation entre répondants, nous avons volontairement apposé à la suite de l'expression "plateformes de vente en ligne " le nom de différentes marketplaces parmi les plus connues et fréquentées par les internautes français. La formulation exacte de la question posée aux répondants a donc été : « Vos produits et/ou services sont-ils déjà disponibles sur une ou plusieurs plateformes de

\footnotetext{
9 Le terme plateforme participe d'une rhétorique de l'intermédiation neutre, de l'existence d'un tiers facilitateur optant pour les appariements économiques les plus opportuns, d'un espace d'exposition et de commensuration d'un vaste ensemble de biens. Or il permet également de faire passer au second plan les "tensions » inhérentes à l'exercice de gestion de ce type d'interface qui doit satisfaire aux attentes de l'ensemble de ses usagers (vendeurs et acheteurs compris) tout en conduisant, dans le même temps, des opérations marketing ciblées attribuant aux plus offrants différents avantages commerciaux. Ainsi, le choix de ce terme « importe autant pour ce qu'il cache que pour ce qu'il révèle » (Gillespie, 2010, p. 359 ; traduction libre de : " these terms matter as much for what they hide as for what they reveal »).
} 
vente en ligne (Amazon, eBay, Leboncoin.fr, AirBnB, etc.) ?". Cette question simple associant l'expression française à une poignée d'exemples emblématiques nous semble avoir trouvé écho auprès des entrepreneurs et n'a posé aucun problème de compréhension de leur part.

Un peu plus loin dans le questionnaire, nous avons demandé aux entrepreneurs s'ils seraient ou non intéressés par un projet de marketplace locale initié par la communauté d'agglomération du Grand Albigeois. Le qualificatif « local » doit ici être compris comme désignant le périmètre restreint de la communauté d'agglomération ${ }^{10}$. Les répondants se prononcent donc en fonction d'une aire commerciale précisément circonscrite. Pour autant, une double incertitude demeure: les entrepreneurs interrogés ignorent d'une part les conditions pratiques d'adhésion à la plateforme (coût d'entrée, modèle économique, entité responsable du pilotage de la marketplace) et, d'autre part, les critères de sélection des offres éligibles (définition du périmètre de production, qualités des biens et services proposés, modes de distribution des biens). Ils se positionnent donc sur un projet encore largement indéfini qui consiste en l'appropriation d'un outil caractéristique du marché global (la plateforme) retravaillé en vue d'augmenter le volume des échanges économiques locaux (relocalisation) ${ }^{11}$. Dans le questionnaire, nous avons arrêté la formulation suivante: "Si une plateforme électronique de vente était créée dans l'agglomération albigeoise de façon à assurer la vente de produits et services locaux, seriezvous intéressé(e) par cette initiative? ». Cette formulation, suffisamment précise pour désigner le projet de départ, mais encore trop lâche pour en faire une juste description, porte en elle le risque d'une surdéclaration de l'intérêt pour la plateforme locale.

Si les répondants disposent d'aussi peu d'informations sur les contours de la plateforme locale, c'est que ses instigateurs ont souhaité, dans la phase inaugurale du développement de leur projet, s'inscrire dans un dialogue constructif avec les

\footnotetext{
10 La communauté d'agglomération du Grand Albigeois regroupe 17 communes et occupe une superficie de 208,9 km². En 2015, elle accueillait 82435 habitants.

11 Nous sommes ici confrontés à une forme d' "infrastructuralisation » de l'objet plateforme (Plantin et al., 2016), c'est-à-dire à la conversion de cet outil caractéristique du marché global (la plateforme) en un dispositif (semi-)public propre à servir le développement durable de l'économie locale, le développement plus transparent et démocratique de l'espace marchand (infrastructure).
} 
Variations autour de l'adoption de plateformes de vente en ligne : la réception d'un nouveau dispositif marchand par les entrepreneurs albigeois

entrepreneurs du territoire et sonder leurs besoins. Ils ont donc souhaité laisser un grand nombre d'options ouvertes. Selon les responsables intercommunaux, quelques principes doivent pour autant guider la conception de cette plateforme. Celle-ci doit en effet contribuer au renforcement de différents programmes d'action économique portés par l'agglomération tels la montée en compétences des entrepreneurs du territoire dans les domaines informatique et numérique, le montage et la promotion de circuits courts, l'aide au développement des entreprises innovantes du Grand Albi.

\section{Encadré 1 : Genèse du projet de marketplace locale}

Initié par le responsable du développement économique de la communauté d'agglomération du Grand Albigeois, le projet de création d'une "marketplace locale» s'intègre au volet numérique du programme "Objectif Business Agglo 2030 ». Le descriptif du projet, tel que restitué dans ce document-cadre, est riche d'enseignements :

"Préfigurations de la mise en œuvre d'une "marketplace" jouant un rôle de mise en relation entre un marché local de 85000 consommateurs et ses 2000 vendeurs. La place de marché est une réponse à plusieurs orientations communautaires à l'échelle [du territoire] :

- Développer une culture du numérique chez les professionnels et les particuliers

- Favoriser les circuits courts

- Mettre en œuvre des solutions innovantes pour que l'argent s'investisse en local, que les initiatives en lien avec l'innovation puissent disposer d'une capacité à trouver un (premier) marché en local

- Étudier un possible développement de l'économie collaborative à l'échelle locale, en portant une attention particulière à l'absence d'opposition frontale par rapport aux circuits traditionnels ».

La feuille de route ici dressée prévoit la mise en place d'un ensemble choral de mesures qui viendraient au soutien d'une économie locale dont la croissance reste fragile. La marketplace serait au cœur d'une refonte d'un ensemble de pratiques productives, commerciales, consuméristes qui tendraient progressivement à toutes converger vers un développement durable et responsable de l'économie. Elle servirait ainsi d'utile point d'appui à l'établissement de circuits courts, à la mise en lumière d'initiatives innovantes pensées par et pour les acteurs locaux et/ou tournées vers le développement d'une «économie collaborative à l'échelle locale ». 
Les responsables de la communauté d'agglomération doivent, sous peu, arrêter les grandes lignes de ce dispositif inédit, établir les conditions de vente régissant son usage (commission ou forfait), délimiter précisément les zones de production et de chalandise retenues (mairie, agglomération, département), penser les supports logistiques associés à cette démarche (consignes, points relais, acheminement des biens par différents modes de transports doux) et désigner l'entité responsable de la gestion de la plateforme (acteur public, délégation de service public ou acteur privé). Pour ce faire, ils ont choisi de conduire une consultation la plus large possible des entreprises du secteur et des acteurs institutionnels locaux chargés des questions économiques. Cette consultation a pour partie été confiée aux deux auteurs du présent article dans le cadre d'une convention de recherche passée entre I'Institut National Universitaire Champollion (INUC) et la communauté d'agglomération. Les responsables de l'agglomération envisagent à présent de faire réaliser une étude d'opportunité par un cabinet de conseil pour avoir une idée plus précise du rendement éventuel d'un tel dispositif, de son impact sur le dynamisme économique local.

C'est dans le cadre du projet de recherche commandité par la communauté d'agglomération que nous avons mis en place, avec le concours des étudiants du Master 2 «Territoires Innovations, Collectivités, Sociétés ${ }^{12}$ ", une enquête quantitative dont l'objectif était d'aller à la rencontre des entrepreneurs du territoire de façon à connaître leurs pratiques en matière de commerce électronique et à sonder leur intérêt pour ce projet de marketplace locale. Qui sont les entrepreneurs que nous avons rencontrés ? Comment se compose notre échantillon?

\subsection{Description de l'échantillon}

La construction de notre échantillon a répondu à une seule et unique exigence: donner la parole à différentes familles d'entrepreneurs de façon à mettre en évidence les variations inhérentes aux conditions d'exercice rapportées et aux intérêts particuliers présidant à l'action des responsables interrogés. Les 193 répondants sont le plus souvent les dirigeants d'entreprise eux-mêmes $(79,1 \%$ des cas) ou leurs délégués qui, en l'absence de leur employeur, se proposaient de nous répondre (responsable d'équipe, chef de projet, DRH). Les réponses recueillies sont donc celles d'individus tenus au courant de la

\footnotetext{
12 Nous remercions Y. Boulanger, J. R. Granero Gallego, L. Mainer Til, P. A. Thioub, $\mathrm{M}$. Savin et $\mathrm{F}$. Vair Piova pour leur contribution à ce travail d'enquête.
} 
Variations autour de l'adoption de plateformes de vente en ligne : la réception d'un nouveau dispositif marchand par les entrepreneurs albigeois

situation économique de l'entreprise et des stratégies de développement envisagées par les équipes dirigeantes. Pour désigner ces individus, nous ferons référence aux " responsables d'entreprises » ou aux " entrepreneurs », ce qui recouvre, à nos yeux, l'ensemble des personnes suffisamment haut placées dans l'organigramme de leur entreprise pour en connaître le fonctionnement et pour être en mesure d'intervenir sur son développement.

Les entreprises de notre échantillon sont toutes implantées dans l'aire urbaine d'Albi et 95,3\% d'entre elles se situent dans le périmètre plus restreint de l'unité urbaine d'Albi. C'est à partir des données dont nous disposons sur ces deux échelles de territoire que nous fonderons la comparaison de notre échantillon avec la population mère des entreprises du territoire considéré (tableau 1). 


\section{Cédric CALVIGNAC et Mathieu VIDAL}

Tableau 1. Comparaison de la distribution sectorielle des entreprises de l'échantillon, de l'unité urbaine et de l'aire urbaine d'Albi

\begin{tabular}{|c|c|c|c|c|c|c|}
\hline & $\begin{array}{l}\text { Échantillon retenu } \\
\text { (effectifs) }\end{array}$ & $\begin{array}{l}\text { Échantillon retenu } \\
\text { (en \%) }\end{array}$ & $\begin{array}{l}\text { Unité Urbaine d'Albi } \\
\text { (effectifs) }\end{array}$ & $\begin{array}{l}\text { Unité urbaine d'Albi } \\
\text { (en \%) }\end{array}$ & $\begin{array}{l}\text { Aire Urbaine d'Albi } \\
\text { (effectifs) }\end{array}$ & $\begin{array}{l}\text { Aire Urbaine d'Albi } \\
\text { (en \%) }\end{array}$ \\
\hline AZ - Agriculture, sylviculture et pêche & 6 & $3,1 \%$ & 123 & $4,7 \%$ & 530 & $12,8 \%$ \\
\hline $\begin{array}{l}\mathrm{BE} \text { - Industrie manufacturière, industries } \\
\text { extractives et autres }\end{array}$ & 14 & $7,3 \%$ & 235 & $9 \%$ & 336 & $8,1 \%$ \\
\hline FZ - Construction & 6 & $3,1 \%$ & 337 & $12,9 \%$ & 588 & $14,1 \%$ \\
\hline $\begin{array}{l}\mathrm{GI} \text { - Commerce de gros et de détail, } \\
\text { transports, hébergement et restauration }\end{array}$ & 108 & $56 \%$ & 819 & $31,3 \%$ & 1090 & $26,2 \%$ \\
\hline$J Z$ - Information et communication & 5 & $2,6 \%$ & 66 & $2,5 \%$ & 96 & $2,3 \%$ \\
\hline KZ- Activités financières et d'assurance & 3 & $1,6 \%$ & 67 & $2,6 \%$ & 98 & $2,4 \%$ \\
\hline LZ - Activités immobilières & 5 & $2,6 \%$ & 85 & $3,2 \%$ & 121 & $2,9 \%$ \\
\hline $\begin{array}{l}\text { MN - Activités spécialisées, scientifiques et } \\
\text { techniques ; Services administratifs et de } \\
\text { soutien }\end{array}$ & 15 & $7,8 \%$ & 349 & $13,3 \%$ & 485 & $11,7 \%$ \\
\hline $\begin{array}{l}\text { OQ - Administration publique, } \\
\text { enseignement, santé humaine et action } \\
\text { sociale }\end{array}$ & 10 & $5,2 \%$ & 275 & $10,5 \%$ & 438 & $10,5 \%$ \\
\hline $\begin{array}{l}\text { RU - Autres activités de services (arts, } \\
\text { spectacles, activités récréatives, sportives, } \\
\text { organisations associatives, réparation } \\
\text { d'ordinateurs et de biens personnels, } \\
\text { activités des ménages...) }\end{array}$ & 21 & $10,9 \%$ & 264 & $10,1 \%$ & 374 & $9 \%$ \\
\hline Total & 193 & $100 \%$ & 2620 & $100 \%$ & 4156 & $100 \%$ \\
\hline
\end{tabular}


Variations autour de l'adoption de plateformes de vente en ligne : la réception d'un nouveau dispositif marchand par les entrepreneurs albigeois

Notre échantillon se caractérise tout d'abord par une forte sous-représentation des entreprises appartenant aux secteurs de la construction $(3,1 \%$ de notre échantillon, contre $12,9 \%$ des entreprises de l'unité urbaine d'Albi), des activités spécialisées, scientifiques et techniques $(7,8 \%$ contre $13,3 \%)$ et de l'administration publique, enseignement, santé humaine et action sociale $(5,2 \%$ contre $10,5 \%)$. À l'inverse, on observe une nette surreprésentation des entreprises appartenant au secteur du commerce de gros et de détail, des transports, de l'hébergement et de la restauration (56\% contre $31,3 \%$ ).

Ajoutons que $41,2 \%$ des entreprises de notre échantillon se sont installées avant le 31 décembre 1997 et $61 \%$, avant le 31 décembre 2007. La majorité des entreprises développe donc une activité qui s'inscrit dans un temps long, comme en atteste l'ancienneté importante de beaucoup d'entre elles.

Enfin, les entreprises de l'échantillon accueillent, pour 31,9\% d'entre elles, de 0 à 1 salarié, pour $42,9 \%$ d'entre elles de 2 à 9 salariés, pour $25,1 \%$ d'entre elles 10 salariés ou plus. À ce titre, on observe une nette sous-représentation des entreprises à faible effectif, car, d'après les données de l'Insee, en 2015, $66,1 \%$ des établissements de l'unité urbaine d'Albi n'ont aucun salarié. Cette sous-représentation s'explique en grande partie par notre méthode de recueil des données ; nous avons effectué du porte-à-porte et, par conséquent, avons porté l'essentiel de nos efforts sur les entreprises bénéficiant d'une devanture, d'une vitrine ou manifestant un signe extérieur de présence. Cette même raison peut être invoquée à l'égard de la "coloration tertiaire » de notre échantillon (surreprésentation des commerçants, restaurateurs et hôteliers, notamment). En effet, le porte-à-porte a surtout permis de rendre visite aux professionnels disposés à accueillir le public. Cela constitue un biais de notre enquête qu'il faut bien prendre en considération.

En définitive, les résultats de notre enquête concernent des entreprises localisées dans le périmètre restreint de l'unité urbaine d'Albi (95,3\%), plutôt bien implantées (ancienneté, présence d'une vitrine ou d'un local), de taille modeste (trois quarts comprennent entre 0 à 9 salariés) et appartenant pour une grande part d'entre elles au secteur du « commerce de gros et de détail, des transports, de l'hébergement et de la restauration » $(56 \%)$. 


\section{Déterminants de l'adoption des marketplaces multi-sites}

Parmi les entreprises de notre échantillon, quelles sont celles qui ont d'ores et déjà adopté une (ou des) solution(s) de marketplaces multi-sites ? Quelle place occupe aujourd'hui l'outil marketplace dans le paysage sociotechnique de ces entrepreneurs?

\subsection{Situer l'outil marketplace dans le paysage des dispositifs numériques de vente}

La plupart des répondants indiquent que leur entreprise est d'ores et déjà présente sur Internet (80 \% d'entre eux). Toutefois, cette présence numérique semble, à leurs yeux, inaboutie et encore largement perfectible puisque l'on constate que $72 \%$ des entrepreneurs interrogés se déclarent convaincus du fait que le développement accru de leur présence sur Internet permettrait à leur entreprise d'améliorer encore davantage ses perspectives de croissance. L'adhésion au principe d'un développement numérique des activités commerciales domine donc très largement.

Pour autant, un nombre non négligeable d'entreprises n'ont pas encore véritablement franchi le pas de cette conversion au e-commerce. En effet, une entreprise sur cinq ne bénéficie encore aujourd'hui d'aucune couverture numérique. Les responsables de ces entreprises invoquent plusieurs freins à la numérisation de leur offre, parmi lesquels figurent le manque de temps pour assurer une présence minimale sur Internet $(46,3 \%)$, le fait que les produits et services vendus ne se prêtent pas à une présence sur Internet $(25,9 \%)$, le fait que la gestion de la présence sur Internet est trop coûteuse (14,8\%) ou encore que les responsables et les employés n'ont pas les connaissances requises pour communiquer sur Internet (13\%). Retenons ici que les non-adoptants des dispositifs numériques de vente sont la plupart du temps empêchés par le coût temporel et (donc) économique de l'adoption de ces solutions, un coût d'adoption qui fluctue, de manière sensible, d'un outil ou d'un service à un autre. Ainsi, on constate que les créations de sites Internet comme les ouvertures de comptes sur différents réseaux sociaux sont aujourd'hui très largement pratiquées, car elles sont devenues simples, intuitives et rapides. Par contre, d'autres 
Variations autour de l'adoption de plateformes de vente en ligne : la réception d'un nouveau dispositif marchand par les entrepreneurs albigeois

outils - dont les plateformes de vente - vont être moins souvent mobilisés parce qu'ils requièrent un investissement temporel plus conséquent (tableau 2).

Tableau 2. Diffusion des outils et services numériques

Taux de diffusion des différents outils et services numériques

\begin{tabular}{|l|c|}
\hline Site Internet de l'entreprise & $87,1 \%$ \\
\hline Présence sur les réseaux sociaux & $78,5 \%$ \\
\hline $\begin{array}{l}\text { Référencement sur des annuaires professionnels en ligne } \\
\text { (au-delà des Pages Jaunes) }\end{array}$ & $69,9 \%$ \\
\hline $\begin{array}{l}\text { Présence sur une plateforme de vente en ligne } \\
\text { (Amazon, eBay, Leboncoin.fr) }\end{array}$ & $26,3 \%$ \\
\hline Achat d'espaces publicitaires sur Internet & $22,2 \%$ \\
\hline Création d'un blogue, animation d'un forum & $18,8 \%$ \\
\hline
\end{tabular}

La plupart des entreprises fondent aujourd'hui leur activité numérique sur la mise en place d'un site Internet dédié, généralement associé à une ou plusieurs pages de présentation de l'entreprise sur des réseaux sociaux populaires (Facebook, Linkedln) ou des annuaires professionnels en ligne. Si l'on se penche sur les principaux types d'association entre dispositifs (sur la base des relevés de fréquence de ces assemblages), on observe que $25,9 \%$ des associations observées relèvent de la combinaison "site + réseau social + référencement annuaire professionnel », $16,3 \%$ de l'association entre un site et un réseau social et $8,9 \%$ de l'association entre un site et un référencement sur un (ou des) annuaire(s) professionnel(s). L'usage cumulé de ces deux à trois solutions courantes est donc très répandu. Ces différentes combinaisons, lorsqu'elles sont bien pensées et configurées, peuvent former un espace de visibilité important pour l'entreprise. Qui plus est, le coût de cette démarche reste relativement modeste puisqu'il se résume au seul coût d'installation et de configuration du site Internet dédié et au seul temps d'inscription au réseau social ou à l'annuaire professionnel visé.

Les démarches plus engageantes consistant à adhérer à une plateforme de vente en ligne, à acheter des espaces publicitaires ou encore à animer un forum ou un espace d'échange avec sa clientèle sur Internet sont beaucoup moins répandues. Elles 
donnent lieu à des dépenses plus importantes et à une supervision continue (relever les commandes, mettre à jour les annonces, sélectionner les régies, les agences, les espaces publicitaires, dialoguer avec la clientèle). Or les entreprises ne sont pas toutes égales devant les investissements en temps et en moyens dévolus au e-commerce et à la communication sur Internet (Mallard, 2011). Seules les plus grandes ont la capacité de créer des postes spécialisés dans ces domaines. Ainsi, et de façon générale, la proportion d'entreprises de 10 salariés ou plus recourant à des outils et des services numériques est sensiblement plus élevée que la moyenne (tableau 3).

Tableau 3. Diffusion des dispositifs e-commerce selon la taille de l'entreprise

\begin{tabular}{|c|c|c|c|}
\hline Utilisation de différents outils et services numériques & $\begin{array}{l}0 \text { à } 1 \\
\text { salarié }\end{array}$ & $\begin{array}{l}2 \text { à } 9 \\
\text { salariés }\end{array}$ & $\begin{array}{l}\geq 10 \\
\text { salariés }\end{array}$ \\
\hline Site Internet de l'entreprise & $76,1 \%$ & $87,5 \%$ & $97,7 \%$ \\
\hline Présence sur les réseaux sociaux & $69,8 \%$ & $75,8 \%$ & $90,5 \%$ \\
\hline $\begin{array}{l}\text { Référencement sur des annuaires professionnels en } \\
\text { ligne }\end{array}$ & $62,5 \%$ & $75,4 \%$ & $69,8 \%$ \\
\hline Présence sur une plateforme de vente en ligne & $35,9 \%$ & $17,9 \%$ & $29,3 \%$ \\
\hline Achat d'espaces publicitaires sur Internet & $26,3 \%$ & $17,9 \%$ & $25 \%$ \\
\hline Création d'un blogue, animation d'un forum & $8,3 \%$ & $19 \%$ & $28,9 \%$ \\
\hline
\end{tabular}

Derrière cette tendance générale se cachent des stratégies singulières d'utilisation des différents outils et services numériques. On constate, par exemple, que $35,9 \%$ des entreprises de 0 à 1 salarié sont présentes sur les plateformes de vente en ligne. Ces très petites entreprises, qui peinent parfois à accéder aux circuits de distribution classiques, se reportent plus volontiers sur les marketplaces dont l'accès est aisé et ne demande pas de bénéficier au préalable d'un capital relationnel minimal ${ }^{13}$. De la même manière, $26,3 \%$ des entreprises de 0 à 1 salarié acquièrent des espaces publicitaires sur Internet de façon à susciter une adhésion rapide à leur offre et, une fois encore, à ne pas avoir à bénéficier du soutien des grands acteurs de la distribution.

\footnotetext{
13 «En contournant les gatekeepers du commerce classique, des activités artistiques et artisanales, de la cartographie, de la cuisine, de l'hébergement ou de la mobilité, des individus ordinaires peuvent se faire connaître et trouver un public » (Beuscart, Flichy, 2018, p. 15).
} 
Variations autour de l'adoption de plateformes de vente en ligne : la réception d'un nouveau dispositif marchand par les entrepreneurs albigeois

Plus généralement, on constate que l'affectation des investissements humain et temporel en vue d'une numérisation des pratiques commerciales est très largement dépendante de la taille des entreprises : les plus grandes jouissent d'un large portefeuille d'outils et de services $(72,7 \%$ des entreprises de 10 salariés ou plus utilisent 3 à 6 dispositifs), alors que les plus petites développent un nombre d'options de communication commerciale plus limité et s'y tiennent $(56,3 \%$ des entreprises de 0 à 1 salarié utilisent 2 dispositifs ou moins) (tableau 4).

Tableau 4. Nombre de dispositifs numériques mobilisés en fonction de la taille de l'entreprise ${ }^{14}$

De 0 à 2 dispositifs De 3 à 6 dispositifs

\begin{tabular}{|l|l|l|}
\hline 0 à 1 salarié & $56,3 \%$ & $43,7 \%$ \\
\hline 2 à 9 salariés & $44,6 \%$ & $55,4 \%$ \\
\hline$\geq 10$ salariés & $27,3 \%$ & $72,7 \%$ \\
\hline
\end{tabular}

Retenons que les marketplaces multi-sites ont été adoptées par un peu plus d'un quart des entreprises de notre échantillon. Ces entreprises peuvent être classées en deux principales catégories : d'une part, les très petites entreprises qui vont faire de cet outil un point de gravité de leur activité commerciale et ne vont que très peu développer les solutions classiques de communication numérique (sites, référencement, réseau social) ; d'autre part, les entreprises de plus grande taille qui vont faire de l'adoption de la plateforme un axe de développement complémentaire à leur activité commerciale sur Internet (ici les dispositifs adoptés sont multiples et peuvent ou non former système).

\subsection{Définir la population des adoptants de marketplaces multi-sites}

II convient à présent de mettre au jour ce qui sépare les $26,3 \%$ d'entreprises ayant adopté une (ou des) marketplace(s) multi-sites du reste des entreprises de notre échantillon. Quels facteurs faut-il retenir pour discriminer cette population, pour faire surgir les traits singuliers qui la caractérisent? Afin d'identifier ces facteurs, nous nous reposerons sur une revue de

${ }^{14} x^{2}=7,928 ; d d l=2 ;$ Sign. $=0,019$. 
littérature ciblée de travaux de sociologie et d'économie ayant spécifiquement traité de l'adoption des technologies numériques en entreprise (e-business et e-commerce confondus). Nous ferons la synthèse des facteurs d'adoption évoqués de façon à en interroger la validité et la pertinence pour le cas qui nous occupe (voir encadré 2 et tableau 5).

\section{Encadré 2 : Revue de littérature et sélection des variables explicatives}

Rares sont les travaux qui ont spécifiquement porté sur l'adoption des marketplaces. La plupart des références ci-après mobilisées portent par conséquent sur l'adoption d'autres technologies numériques (sites Internet, système d'échange de données informatisées, adhésion à des annuaires et des réseaux professionnels, etc.). Elles donnent à voir les déterminants communs à la diffusion de différentes générations de technologies de l'information et de la communication (TIC) qui ont contribué, à des degrés variables, au mouvement général d'électronisation du commerce. Nous classerons les variables repérées dans la littérature en trois principales catégories: les variables structurelles (niveau macro: politiques nationales, secteur d'activités, réseaux de distribution, niveau de concurrence), organisationnelles (niveau méso: l'entreprise, ses caractéristiques et ses stratégies commerciales) et individuelles (niveau micro: l'entrepreneur et ses compétences).

\section{Variables structurelles}

Parmi les variables structurelles sont souvent évoquées: l'importance des grandes orientations nationales en matière de développement technologique, les configurations nationales (et internationales) des principaux réseaux de distribution des biens, le niveau général d'alphabétisation numérique des populations. La considération de ce premier ensemble de facteurs a ainsi donné lieu à la réalisation de différentes enquêtes fondées sur une approche comparatiste et interculturelle particulièrement riche d'enseignements (Aoyama, 2003 ; Brousseau, 2003 ; Brousseau et Chaves, 2004). Ce premier niveau d'explication - qui traite de l'influence des grands investissements technologiques et infrastructurels consentis aux échelles nationale ou internationale - dépasse de loin nos objectifs de recherche. De plus, ils ne peuvent discriminer les entreprises de notre échantillon qui sont toutes soumises aux mêmes conditions nationales d'exercice. Elles ne figureront donc pas dans notre modèle.

En revanche, un autre ensemble de variables structurelles peut être utilement intégré à notre enquête. II s'agit des variables se rapportant à l'appartenance sectorielle de l'entreprise, au taux de diffusion des TIC chez les principaux partenaires industriels et commerciaux de 
Variations autour de l'adoption de plateformes de vente en ligne : la réception d'un nouveau dispositif marchand par les entrepreneurs albigeois

l'entreprise (fournisseurs, consultants, clients), mais aussi au niveau de
pression concurrentielle auquel les entrepreneurs déclarent être
confrontés (Amabile et Gadille, 2000; Beuscart, Dagiral, Parasie,
2016 ; Gadille et d'Iribarne, 2000 ; Hong et Zhu, 2005; Lin et Lin, 2008;
Mallard, 2011 ;Oliveira et Martins, 2010a, 2010b; Windrum et de
Berranger, 2002 ; Zhu, Kraemer et Xu, 2003). Différents chercheurs ont
observé que les chances de voir une entreprise adopter un (ou des)
dispositif(s) numérique(s) de vente augmentent lorsque celle-ci est
soumise à une intense concurrence, lorsque le secteur d'activités
auquel elle appartient se restructure fortement autour du e-commerce
et lorsque ses principaux partenaires industriels et commerciaux sont
déjà équipés. Ces différents facteurs peuvent tous être ramenés à un
même phénomène : l'importance des effets d'externalités opérant sur
la diffusion des TIC (Arthur, 1989). Une fois un certain seuil d'adoption
franchi dans l'entourage professionnel de l'entreprise (partenaires,
concurrents, clients), les effets d'externalités de réseau jouent à plein
et favorisent une accélération de la diffusion des solutions portées. Ces
trois facteurs (secteur d'activités, taux d'équipement des partenaires et
concurrence ressentie) sont intégrés à notre modèle. Nous y ajoutons
une dernière variable : celle de l'affiliation (ou non) à un réseau
d'entreprises franchisées. II nous semble en effet judicieux de distinguer
indépendants et franchisés, car ils ne jouissent pas des mêmes libertés
en matière d'équipement technologique, mais aussi de définition des
politiques de communication et des stratégies commerciales à conduire
(Dubuisson-Quellier, 2003).

\section{Variables organisationnelles}

Mettons à présent l'accent sur l'entreprise : ses caractéristiques, ses activités, ses principaux axes de développement. Considérons l'importance relative de trois premières variables organisationnelles: l'ancienneté, la taille et la localisation de l'entreprise. L'ancienneté de l'entreprise fait l'objet d'une faible attention dans les travaux consultés (Jullien et Trémembert, 2007). Nous l'intégrons ici de façon à éprouver I'hypothèse selon laquelle les entreprises les plus anciennement installées auraient davantage de difficultés et de moins grands avantages à se convertir au e-commerce, là où les nouveaux entrants auraient plus de facilité et d'intérêt à en concevoir un usage plus immédiat et intensif. La deuxième variable intégrée $a$, au contraire, fait l'objet d'une très grande attention de la part des sociologues et des économistes. II s'agit du critère de la taille de l'entreprise. La plupart des travaux s'accordent à dire que l'effet taille est ambivalent. En effet, on observe généralement qu'au premier temps de la diffusion des technologies e-commerce, les grandes entreprises sont mieux placées que les TPE/PME, car elles disposent d'une capacité d'investissement supérieure permettant d'investir à la fois dans le matériel informatique, dans la conception d'applications logicielles ad hoc et dans l'affectation de ressources humaines dédiées à l'amélioration et au maintien des systèmes informatiques du groupe (Jullien et Trémembert, 2007 ; Hong 
et Zhu, 2005 ; Windrum et de Berranger, 2002). Dans un second temps, la situation peut s'inverser au point de voir les TPE/PME revenir au niveau des plus grandes, voire les dépasser à certains égards dans l'utilisation des technologies e-commerce. En effet, une fois que la baisse des coûts d'adoption le leur permet, les TPE/PME sont plus agiles et davantage enclines à faire leurs ces nouvelles technologies. De plus, elles ne sont pas soumises aux mêmes effets d'inertie que les plus grandes. Les grandes entreprises, parce qu'elles font appel à un réseau d'acteurs plus important (qu'il s'agit de coordonner de façon efficace et qui ont besoin, pour être performants, de modes de communication et d'échanges d'information stabilisés), ont en effet davantage de difficultés à migrer d'un système à un autre, d'une technologie à une autre (Cochoy, 2003 ; Hong et Zhu, 2005 ; Oliveira et Martins, 2010a, 2010b ; Zhu, Kraemer et Xu, 2003). Concernant l'emplacement des entreprises, troisième variable organisationnelle intégrant notre modèle, nous avançons l'hypothèse selon laquelle l'éloignement des grands axes de transports et des principales aires commerciales d'un territoire rendent plus impérieux le besoin de souscrire à des technologies e-commerce.

D'autres variables organisationnelles sont à prendre en considération, notamment celles se rapportant à la façon dont les entrepreneurs échafaudent de nouvelles stratégies commerciales, mettent en place de nouvelles méthodes de "captation des publics" (Cochoy, 2004). C'est notamment le cas lorsque les entrepreneurs font d'Internet une fenêtre de communication propre à venir pallier l'absence de vitrine (Mallard, 2011) ou de locaux commerciaux (bureau, boutique). C'est également le cas lorsque les entrepreneurs décident d'étendre leur zone de chalandise, d'atteindre des clients lointains (géographiquement parlant) ou distants (hors des circuits de diffusion traditionnels de l'information commerciale). C'est enfin le cas lorsque des entrepreneurs dont l'activité est en perte de vitesse (baisse ou stagnation de l'activité) décident de miser davantage sur le e-commerce pour mieux rebondir. Ces trois variables organisationnelles (effet vitrine, extension de la zone de prospection/chalandise et situation économique de l'entreprise) sont intégrées au modèle.

Un dernier ensemble de variables organisationnelles porte quant à lui sur les propriétés des biens et services offerts et sur les conditions de leur production. II sera question d'interroger les stratégies de valorisation de la dimension locale de l'offre. Deux questions ont été adressées aux répondants en ce sens: l'une portant sur l'ancrage territorial de la production (emploi de ressources locales), l'autre sur la volonté de développer des circuits courts.

\section{Variables individuelles}

Enfin, sur le plan des variables individuelles, l'attention des chercheurs s'est particulièrement portée sur la relation entre la pénétration de la technologie dans la sphère privée et dans la sphère 
Variations autour de l'adoption de plateformes de vente en ligne : la réception d'un nouveau dispositif marchand par les entrepreneurs albigeois

professionnelle. Cette relation est réciproque: la maîtrise d'une technologie incite les acteurs à en déplacer l'usage et à bénéficier des avantages qu'il procure d'une sphère à l'autre. Sur cette question, Alexandre Mallard indique que, dans les petites entreprises, la perméabilité entre sphères personnelle (activité numérique pratiquée sur son temps libre) et professionnelle (activité pratiquée dans l'exercice de son métier) est plus importante que dans les grandes (Mallard, 2011; Pierson, 2005). II précise que les très petites entreprises sont plus que les autres dépendantes des ressources et compétences de leur dirigeant. Deux variables de contrôle seront intégrées à notre modèle pour le vérifier : l'aisance des entrepreneurs vis-à-vis des TIC et leur niveau de diplôme.

Tableau 5. Variables mobilisées pour discriminer les entreprises adoptantes

\begin{tabular}{|c|c|c|c|}
\hline & Variables explicatives & Hypothèses de recherche & Références \\
\hline \multicolumn{4}{|c|}{ Variables structurelles } \\
\hline 1 & Secteur d'activités & $\begin{array}{l}\text { Nature des biens ou des } \\
\text { services échangés } \\
\text { Réseaux de distribution } \\
\text { Relation aux partenaires } \\
\text { commerciaux } \\
\text { Externalités de réseau }\end{array}$ & $\begin{array}{l}\text { Amabile, Gadille, } 2000 \\
\text { Gadille, d'Iribarne, 2000, } \\
\text { Windrum, de Berranger, } 2002 \\
\text { Aoyama, } 2003 \\
\text { Brousseau, } 2003 \\
\text { Zhu, Kraemer, Xu, } 2003 \\
\text { Brousseau, Chaves, } 2004 \\
\text { Hong, Zhu, } 2005 \\
\text { Jullien, Trémembert, } 2007 \\
\text { Lin, Lin, } 2008 \\
\text { Oliveira, Martins, 2010a, 2010b } \\
\text { Mallard, } 2011\end{array}$ \\
\hline 2 & $\begin{array}{l}\text { Affiliation à un réseau ou à } \\
\text { un collectif } \\
\text { d'entrepreneurs }\end{array}$ & $\begin{array}{l}\text { Liens préexistants avec } \\
\text { différents collectifs } \\
\text { Structuration des échanges } \\
\text { économiques locaux }\end{array}$ & $\begin{array}{l}\text { Gadille, d'Iribarne, 2000, } \\
\text { Dubuisson-Quellier, } 2003 \\
\text { Jullien, Trémembert, } 2007 \\
\text { Lin, Lin, } 2008 \\
\text { Oliveira, Martins, 2010a, 2010b }\end{array}$ \\
\hline 3 & $\begin{array}{l}\text { Secteur } \\
\text { hautement/faiblement } \\
\text { concurrentiel }\end{array}$ & $\begin{array}{l}\text { Caractère urgent de l'adoption } \\
\text { Effets d'anticipation } \\
\text { Stratégies de mimétisme- } \\
\text { différenciation }\end{array}$ & $\begin{array}{l}\text { Gadille, D'Iribarne, 2000, } \\
\text { Zhu, Kraemer, Xu, } 2003 \\
\text { Lin, Lin, } 2008 \\
\text { Oliveira, Martins, 2010a, 2010b } \\
\text { Mallard, } 2011\end{array}$ \\
\hline 4 & $\begin{array}{l}\text { Franchisés ou } \\
\text { indépendants }\end{array}$ & $\begin{array}{l}\text { Liberté de choix versus } \\
\text { alignement }\end{array}$ & Dubuisson-Quellier, 2003 \\
\hline \multicolumn{4}{|c|}{ Variables organisationnelles } \\
\hline 5 & $\begin{array}{l}\text { Date d'installation de } \\
\text { l'entreprise }\end{array}$ & Critère d'ancienneté & $\begin{array}{l}\text { Ayoama, } 2003 \\
\text { Jullien, Trémembert, } 2007\end{array}$ \\
\hline 6 & Localisation de l'entreprise & $\begin{array}{l}\text { Affiliation territoriale } \\
\text { Centralité de l'emplacement }\end{array}$ & $\begin{array}{l}\text { Windrum, de Berranger, } 2002 \\
\text { Ayoama, } 2003 \\
\text { Dubuisson-Quellier, } 2003 \\
\text { Jullien, Trémembert, } 2007\end{array}$ \\
\hline 7 & Taille de l'entreprise & $\begin{array}{l}\text { Capacités d'investissement } \\
\text { Division du travail } \\
\text { Spécialisation des tâches }\end{array}$ & $\begin{array}{l}\text { Amabile, Gadille, } 2000 \\
\text { Gadille, D'Iribarne, 2000, } \\
\text { Cochoy, } 2003 \\
\text { Dubuisson-Quellier, } 2003 \\
\text { Zhu, Kraemer, Xu, } 2003 \\
\text { Hong, Zhu, 2005 } \\
\text { Jullien Trémembert, } 2007 \\
\text { Mallard, } 2011\end{array}$ \\
\hline 8 & $\begin{array}{l}\text { Clientèle touristique ou } \\
\text { non }\end{array}$ & $\begin{array}{l}\text { Captation de publics distants } \\
\text { Identification des publics cibles }\end{array}$ & $\begin{array}{l}\text { Cardon, } 2015 \\
\text { Beuscart et al., } 2016 \\
\end{array}$ \\
\hline
\end{tabular}




\begin{tabular}{|c|c|c|c|}
\hline 9 & $\begin{array}{l}\text { Volonté d'élargir sa zone } \\
\text { de chalandise }\end{array}$ & $\begin{array}{l}\text { Ouverture à de nouveaux clients } \\
\text { Politique de captation de la } \\
\text { clientèle } \\
\text { Stratégies d'expansion }\end{array}$ & $\begin{array}{l}\text { Amabile, Gadille, } 2000 \\
\text { Cochoy, } 2003 \\
\text { Dubuisson-Quellier, } 2003 \\
\text { Lin, Lin, } 2008 \\
\text { Oliveira, Martins, 2010a,2010b } \\
\text { Mallard, } 2011\end{array}$ \\
\hline 10 & $\begin{array}{l}\text { Existence ou non d'une } \\
\text { vitrine d'exposition }\end{array}$ & $\begin{array}{l}\text { Observation d'un effet vitrine } \\
\text { Affichage et publicisation de } \\
\text { l'offre } \\
\text { Mise en visibilité de l'entreprise }\end{array}$ & $\begin{array}{l}\text { Dubuisson-Quellier, } 2003 \\
\text { Mallard, } 2011\end{array}$ \\
\hline 11 & $\begin{array}{l}\text { Croissance, stabilisation, } \\
\text { diminution de l'activité }\end{array}$ & Opportunités, Effets d'aubaine & Non traité \\
\hline 12 & $\begin{array}{l}\text { Volonté d'adhérer à un } \\
\text { circuit court }\end{array}$ & $\begin{array}{l}\text { Modalités de distribution de son } \\
\text { offre }\end{array}$ & Non traité \\
\hline 13 & $\begin{array}{l}\text { Entreprises valorisant les } \\
\text { ressources locales }\end{array}$ & $\begin{array}{l}\text { Importance des ressources } \\
\text { locales } \\
\text { Identification territoriale des } \\
\text { offres }\end{array}$ & Non traité \\
\hline \multicolumn{4}{|c|}{ Variables individuelles } \\
\hline 14 & $\begin{array}{l}\text { Aisance des entrepreneurs } \\
\text { vis-à-vis des TIC }\end{array}$ & $\begin{array}{l}\text { Capital technique des } \\
\text { entrepreneurs }\end{array}$ & $\begin{array}{l}\text { Dubuisson-Quellier, } 2003 \\
\text { Pierson, } 2005 \\
\text { Jullien, Trémembert, } 2007 \\
\text { Mallard, } 2011\end{array}$ \\
\hline 15 & $\begin{array}{l}\text { Dernier diplôme de } \\
\text { l'entrepreneur }\end{array}$ & $\begin{array}{l}\text { Capital scolaire des } \\
\text { entrepreneurs }\end{array}$ & Suggéré mais non traité \\
\hline
\end{tabular}

Afin d'établir la contribution de chacune de ces variables à l'explication du phénomène, nous avons tout d'abord réalisé une série d'analyses bivariées qui ont permis de mettre en évidence cinq variables significativement corrélées au fait d'adopter (ou non) une marketplace multi-sites ( $p \leq 0,05)$. Puis, et de façon à neutraliser d'éventuels effets de composition entre ces variables, nous avons effectué une régression logistique binaire (modèle Logit). Le modèle finalement retenu ne se compose plus que de trois variables qui, chacune, se sont avérées être significativement corrélées - cette fois toutes choses étant égales par ailleurs - au fait d'adopter (ou non) une marketplace multi-sites. II s'agit du statut de l'entreprise (franchisé ou indépendant), du niveau d'études de l'entrepreneur et de sa volonté (ou non) d'étendre sa zone de chalandise (tableau 6). 
Variations autour de l'adoption de plateformes de vente en ligne : la réception d'un nouveau dispositif marchand par les entrepreneurs albigeois

Tableau 6. Adoption d'une marketplace multi-sites - Contribution des différents facteurs ${ }^{15}$

$\begin{array}{llll}\text { Produits/services déjà } & \text { Coefficient } & \text { Odds ratio } & \text { Significativité } \\ \text { présents sur une } & {[B]} & {[\operatorname{Exp}(B)]} & \end{array}$

marketplace multi-sites ${ }^{a}$

\begin{tabular}{|l|l|l|l|}
\hline Oui $\begin{array}{l}\text { Niveau d' études : } \\
\text { dans le supérieur }\end{array}$ & 1,375 & 3,957 & 0,006 \\
$\begin{array}{l}\text { Niveau d'études : } \\
\text { inférieur ou éq. Bac }\end{array}$ & $0^{\mathrm{b}}$ & & \\
\hline $\begin{array}{l}\text { Extension zone de } \\
\text { chalandise : Oui }\end{array}$ & 1,147 & 3,150 & 0,008 \\
\hline $\begin{array}{l}\text { Extension zone de } \\
\text { chalandise : Non }\end{array}$ & $0^{\mathrm{b}}$ & & 0,009 \\
\hline $\begin{array}{l}\text { Statut de l'entreprise : } \\
\text { franchisée }\end{array}$ & 1,528 & 4,607 & \\
\hline $\begin{array}{l}\text { Statut de l'entreprise : } \\
\text { indépendante }\end{array}$ & $0^{\mathrm{b}}$ & & \\
\hline
\end{tabular}

a. La catégorie de référence est: Non

b. Ce paramètre est défini sur 0 , car il est redondant.

Lecture du tableau : La situation de référence (ou constante) se rapporte aux entrepreneurs

dont le niveau d'études est inférieur ou équivalent au baccalauréat, qui ne souhaitent pas

étendre leur zone de prospection/chalandise et dont l'entreprise est indépendante.

Le modèle obtenu démontre que les entrepreneurs à la tête d'entreprises franchisées $(B=1,528, \quad p<0,05)$, les entrepreneurs diplômés du supérieur $(B=1,375, p<0,05)$ et ceux ayant pour objectif d'étendre leur zone de prospection et de chalandise $(B=1,147, p<0,05)$ sont plus susceptibles d'adopter une (ou des) marketplace(s) multi-sites. Regardons à présent de plus près les différences observées pour chacune de ces variables et tentons de livrer quelques éléments d'explication.

\subsubsection{Indépendants versus franchisés}

L'adoption des marketplaces multi-sites est tout d'abord dépendante de l'affiliation (ou non) de l'entreprise à un réseau de franchise. En effet, quand 19,8\% des entreprises indépendantes déclarent avoir adopté une marketplace multisites, $45 \%$ des entreprises franchisées en font autant. II y a donc une probabilité bien plus grande de voir les entreprises franchisées bénéficier de ce dispositif numérique de captation marchande. II faut dire que les entrepreneurs franchisés

15 Informations sur l'ajustement du modèle : $x^{2}=23,706$; ddl = 3 ; Sig. = 0,000. 
s'appuient, en la matière, sur les propositions du franchiseur. Or, ce dernier, parce qu'il centralise et agrège des ressources humaines et techniques importantes, est en mesure de réaliser sa propre plateforme propriétaire ou d'administrer un compte entreprise sur une ou plusieurs plateformes partenaires.

L'examen de ce premier facteur nous rappelle que nombre d'entreprises se placent sous la gouvernance partielle de différents collectifs tels les franchises, les filiales ou les consortiums d'achat. Or l'affiliation à ces (re)groupements pèse de façon considérable sur la définition des stratégies commerciales à suivre. En effet, intégrer un réseau d'entreprises, c'est mettre en partage un savoir-faire commercial et technique plus étendu. Aussi, et avec le concours d'entreprises partenaires ou tutélaires, les franchisés adoptent plus précocement les technologies les plus récentes. Ils se saisissent d'outils généralement préconfigurés par le franchiseur, de solutions clés en main imposées par le haut. On assiste alors à l'avènement d'une logique de conformation corporative qui consacre une forme avancée d'homogénéisation des pratiques.

\subsubsection{Adoption d'une marketplace multi-sites et niveau de diplôme}

Le deuxième facteur à prendre en considération a trait aux qualités et aux compétences des entrepreneurs. Dans la littérature, il est souvent fait référence au fait que l'aisance des individus vis-à-vis des TIC serait positivement associée à leurs chances d'adoption de nouveaux outils numériques. Sans remettre en cause ces analyses, nous voudrions indiquer qu'elles ne s'appliquent pas à notre cas, sans doute parce que l'entrepreneur est davantage contraint - de par la situation concurrentielle qu'il expérimente - de renouveler régulièrement ses techniques de vente. Cela exige de sa part, quelle que soit l'aisance éprouvée, de trouver une solution pour que son offre figure en bonne place sur le marché (notamment sur les marketplaces multi-sites). Les entrepreneurs peu à leur aise avec les TIC peuvent ainsi confier le soin de développer le volet e-commerce de leur entreprise à des employés compétents (en interne) ou à des prestataires de services (sous-traitance). L'aisance des responsables d'entreprise vis-à-vis des TIC n'a donc pas de réelle influence sur les taux d'adoption observés 
Variations autour de l'adoption de plateformes de vente en ligne : la réception d'un nouveau dispositif marchand par les entrepreneurs albigeois

(22\% chez les entrepreneurs déclarant une forte aisance, $22,6 \%$ chez ceux déclarant une aisance faible à modérée).

Par contre, le capital scolaire (mesuré à l'aide du dernier diplôme obtenu) pèse incontestablement sur l'adoption des marketplaces multi-sites. En effet, les entrepreneurs diplômés du supérieur sont $29,3 \%$ à proposer leurs produits et services sur une ou des plateformes de vente en ligne quand ceux ayant un niveau inférieur ou équivalent au baccalauréat ne sont plus que $10,8 \%$ à en faire autant. Ce n'est donc pas sur le plan des compétences ou des habiletés techniciennes que réside la différence d'engagement, mais bien davantage sur celui de la stratégie d'entreprise, de la vue d'ensemble dont bénéficient certains entrepreneurs formés à une meilleure gestion des composantes informationnelle, communicationnelle et marketing de leur activité (économie-discipline). En d'autres termes, la mise en place de ces nouveaux dispositifs de vente n'est pas liée à l'aisance des entrepreneurs vis-à-vis des TIC, mais bel et bien à l'identification des opportunités qu'elles confèrent en ce qui concerne la visibilisation de leur activité. Les plus diplômés passés par des formations universitaires généralement tournées vers les métiers de la vente, de la gestion et du commerce seraient davantage disposés à se saisir de ces nouvelles générations d'outils susceptibles de mieux signaler la singularité de leur offre.

\subsubsection{Adoption d'une marketplace multi-sites et extension de la zone de chalandise}

Le troisième et dernier facteur à prendre en considération tient à la volonté (ou non) d'étendre sa zone de prospection et de chalandise. Si l'on discrimine les entreprises en fonction de ce critère, on constate que les entreprises affirmant poursuivre cet objectif déclarent plus fréquemment que les autres avoir adopté une (ou des) marketplace(s) multi-sites (30,3\% contre $12 \%)$. Ce résultat n'a rien de surprenant : l'usage d'une marketplace multisites dépend, c'était à attendre, de la volonté d'atteindre de nouveaux publics sur lesquels l'entrepreneur n'avait jusqu'alors aucune prise.

Précisons ici que les entrepreneurs adoptent deux types différents de plateformes multi-sites donnant accès à deux clientèles distinctes : d'une part, les marketplaces globales, qui mettent en relation un large contingent d'offreurs et de 
demandeurs sans se soucier ni de leur origine géographique ni de la distance physique qui les sépare (de type Amazon) ; d'autre part, les marketplaces "glocales", qui déclinent un même modèle, une même solution d'intermédiation sur différents territoires de façon à créer les conditions d'une rencontre entre vendeurs et acheteurs d'une même région (de type Leboncoin.fr).

Parmi les 42 répondants ayant déclaré avoir souscrit aux services d'une ou de plusieurs plateformes, 35 ont accepté de préciser quelles étaient les plateformes multi-sites utilisées. Dans l'ensemble, 75 citations ont été dénombrées, ce qui permet d'ores et déjà d'affirmer que l'ouverture de comptes sur plusieurs plateformes (ou multihoming) est une pratique relativement courante. Sur les 75 occurrences recensées, 26 décrivent des plateformes "glocales" et 49 des plateformes "globales". Dans le cas de l'adoption des premières, les entrepreneurs font de la marketplace un révélateur de l'extensibilité du marché local (multitude de voisinage). Dans le cas de l'adoption des secondes, ils perçoivent la marketplace comme un moyen d'accéder à une multitude éclatée dans un espace numérique " sans frontière ".

Nous retiendrons ici que l'adoption des marketplaces multisites est dépendante de l'inscription de l'entreprise dans des réseaux professionnels susceptibles de préétablir les conditions de souscription aux offres d'intermédiation (franchisés vs indépendants), de l'acculturation de l'entrepreneur aux enjeux du e-commerce (dernier diplôme obtenu) et enfin de la volonté de publiciser son offre auprès d'un public plus étendu (multitude éclatée ou multitude de voisinage). Ainsi, l'ensemble des facteurs présidant à l'adoption de marketplaces multi-sites répondent à des logiques d'inscription congruentes : il s'agit de s'inscrire au cœur d'une place marchande et d'en adopter les instruments, de s'inscrire dans des schémas de coopération interentreprises (franchisés vs indépendants), de s'inscrire dans une économie-discipline (dont les fondements sont mieux maîtrisés par les entrepreneurs diplômés du supérieur) dont la « performation » se traduit par une numérisation des moyens de vente afin d'élargir sa clientèle. 
Variations autour de l'adoption de plateformes de vente en ligne : la réception d'un nouveau dispositif marchand par les entrepreneurs albigeois

\section{Déclarations d'intérêt pour une marketplace locale}

Nous allons maintenant quitter les déclarations portant sur l'usage des marketplaces multi-sites pour examiner les déclarations d'intérêt prononcées en faveur de l'adhésion à une marketplace locale. Quels sont les entrepreneurs qui se déclarent intéressés par cette initiative? De nombreuses variables explicatives peuvent peser sur les déclarations d'intérêt enregistrées. Ici, nous reprenons la liste des variables précédemment intégrées à notre modèle explicatif (voir tableau 5) et l'enrichissons de la variable " adoption (ou non) d'une marketplace multi-sites ». Celle-ci permet de contrôler si l'adoption première d'une marketplace multi-sites peut (ou non) favoriser l'adoption seconde d'une marketplace locale.

Commençons par indiquer que $59 \%$ des entrepreneurs interrogés se déclarent intéressés par la marketplace locale. Pour rappel, seulement $26,3 \%$ de ces mêmes entrepreneurs ont adopté une marketplace multi-sites. Ce doublement des effectifs s'explique, à nos yeux, par quatre principaux facteurs dont nous ne pouvons que présumer l'importance relative: le premier de ces facteurs relève de l'inconséquence inhérente à la simple déclaration d'intérêt (les coûts associés à une adhésion fictive sont nuls), le deuxième facteur a trait à l'indéfinition du projet qui ouvre sur une adhésion encore large risquant toutefois de s'étioler à mesure que seront retenus les différents principes de régulation de la plateforme (tarification, sélection des offres) ; le troisième facteur relève de l'assurance que donne un confinement localiste du marché (rétablir une concurrence de proximité, c'est par définition faire face à moins de concurrents) ; le quatrième et dernier facteur est celui de l'identité des porteurs du projet (les responsables de l'agglomération), qui a de quoi rassurer les entrepreneurs qui entretiennent, pour beaucoup, des relations de confiance avec les responsables publics de la communauté d'agglomération du Grand Albigeois (C2A).

Ces quelques réserves ne doivent pas nous empêcher d'examiner l'intérêt manifesté pour la marketplace locale (pour ce qu'il est), car si la déclaration d'intérêt ne vaut pas adoption, il est évident que toute adoption fait suite à la manifestation d'un intérêt. Comme nous l'avons précédemment fait pour expliquer l'adoption effective des marketplaces multi-sites, nous avons 
procédé à une analyse statistique en deux temps: dans un premier temps, nous avons conduit une série d'analyses bivariées (désignation de sept variables significativement corrélées) et, dans un second temps, procédé à une régression logistique binaire (modèle Logit) de façon à neutraliser les effets de composition entre ces variables. Le modèle finalement retenu ne se compose plus que de trois variables qui, chacune, se sont avérées être significativement corrélées - cette fois toutes choses étant égales par ailleurs - au fait de déclarer (ou non) son intérêt pour une marketplace locale. Ces trois variables sont la valorisation (ou non) des ressources locales par l'entreprise, le souhait de s'inscrire dans une démarche de circuit court et la volonté d'élargir sa zone de prospection et de chalandise (tableau 7).

Tableau 7. Intérêt déclaré pour une marketplace locale Contribution des différents facteurs ${ }^{16}$

\begin{tabular}{|c|c|c|}
\hline $\begin{array}{l}\text { Intérêt déclaré pour une } \\
\text { marketplace locale dédiée à la }\end{array}$ & Coefficient [B] & $\begin{array}{l}\text { Odds ratio } \\
{[\operatorname{Exp}(B)]}\end{array}$ \\
\hline
\end{tabular}

vente de produits et services

locaux dans l'Albigeois $(\mathrm{C} 2 \mathrm{~A})^{\mathrm{a}}$

\begin{tabular}{|ll|l|l|l|}
\hline Oui & $\begin{array}{l}\text { Extension zone de } \\
\text { chalandise : Oui }\end{array}$ & 1,065 & 2,900 & 0,002 \\
$\begin{array}{l}\text { Extension zone de } \\
\text { chalandise : Non }\end{array}$ & $0^{\mathrm{b}}$ & & \\
$\begin{array}{l}\text { Intéressé par les circuits } \\
\text { courts : Oui }\end{array}$ & 1,577 & 4,842 & 0,000 \\
$\begin{array}{l}\text { Intéressé par les circuits } \\
\text { courts : Non }\end{array}$ & $0^{\mathrm{b}}$ & & \\
$\begin{array}{l}\text { Coloration locale de } \\
\text { l'offre : Oui }\end{array}$ & 0,803 & 2,233 & 0,024 \\
$\begin{array}{l}\text { Coloration locale de } \\
\text { l'offre : Non }\end{array}$ & $0^{\mathrm{b}}$ & & \\
\hline
\end{tabular}

a. La catégorie de référence est: Non

b. Ce paramètre est défini sur 0 , car il est redondant.

Lecture du tableau : La situation de référence (ou constante) se rapporte aux entrepreneurs n'ayant pas l'intention d'élargir leur zone de prospection/chalandise, déclarant ne pas être intéressés par la mise en place de circuits courts et dont les produits ou services ne sont pas développés localement.

Le modèle obtenu démontre que les entrepreneurs valorisant des ressources locales $(B=0,803, p<0,05)$, ceux favorables à l'avènement de circuits courts $(B=1,577, p<0,05)$ et ceux ayant pour objectif d'étendre leur zone de prospection et de chalandise $(B=1,065, p<0,05)$ sont plus susceptibles de se déclarer

${ }^{16}$ Informations sur l'ajustement du modèle : $x^{2}=37,418$; ddl = 3 ; Sig. $=0,000$. 
Variations autour de l'adoption de plateformes de vente en ligne : la réception d'un nouveau dispositif marchand par les entrepreneurs albigeois

intéressés par le projet de marketplace locale. Revenons sur ces trois éléments pour mieux se figurer leur influence respective sur les chances d'adhérer à ce projet local.

\subsection{Intérêt pour la marketplace locale et exploitation des ressources locales}

Les entrepreneurs à la tête d'entreprises valorisant les ressources locales (humaines ou techniques) sont proportionnellement plus nombreux à affirmer leur intérêt pour la marketplace locale. Quand 67,6\% d'entre eux déclarent être intéressés par l'initiative, seuls $45,6 \%$ des « revendeurs » ou « intermédiaires » en font autant. Ainsi, une part importante des entrepreneurs a bel et bien intégré la nécessité de limiter l'accès à cette nouvelle interface commerciale aux offres porteuses $\mathrm{du}$ plus grand bénéfice économique local.

Le respect de la vocation localiste du projet peut également se lire dans les déclarations des entrepreneurs portant sur la délimitation du périmètre à retenir pour sélectionner les offreurs (site de l'entreprise), mais également les offres (emploi ou non de ressources locales). Sur le premier des deux facteurs, on observe que $72,6 \%$ des répondants pensent que l'accès à la plateforme locale devrait être réservé aux seuls entrepreneurs implantés dans un secteur restreint, secteur qui, pour $46,5 \%$ des répondants, devrait être circonscrit à l'agglomération albigeoise et, pour $41,7 \%$, se limiter au seul département. II faudrait donc que les vendeurs présents sur la plateforme appartiennent au département tarnais. Concernant maintenant la coloration locale des produits et services offerts, c'est-à-dire la mobilisation de ressources locales, les avis sont moins tranchés. En effet, $59 \%$ des répondants considèrent que cette plateforme devrait être réservée aux seuls entrepreneurs qui produisent localement ou qui mettent en place des circuits courts. Parmi les répondants ayant déclaré valoriser les ressources locales $(n=106)$, la préférence accordée aux productions locales est renforcée puisque $67 \%$ d'entre eux considèrent que la plateforme devrait être réservée aux seuls entrepreneurs qui produisent localement ou mettent en place des circuits courts. Ici, on saisit bien la tension existant entre le fait de privilégier les acteurs locaux (offreurs) ou les actions locales (offres). Parmi les entrepreneurs locaux, certains souhaiteraient bénéficier de la marketplace locale, mais ont bien conscience que leurs produits sont en 
grande partie originaires de producteurs situés hors du département. Ces derniers ont donc tendance à prôner une sélection des offreurs en lieu et place d'une sélection des offres.

\subsection{Intérêt pour la marketplace locale et volonté d'adhérer à des circuits courts}

Les entrepreneurs favorables à l'avènement de circuits courts sont $69,3 \%$ à déclarer leur intérêt pour la marketplace locale. Les deux démarches sont, à leurs yeux, bel et bien congruentes. II est vrai que la reconfiguration logistique des filières d'acheminement local pourrait utilement s'appuyer sur des outils numériques de centralisation des commandes et d'organisation des moyens de distribution, ce que permettrait la plateforme. Quant aux entrepreneurs qui, au contraire, se tiennent à distance de la démarche des circuits courts, ils ne sont plus que $27,5 \%$ à manifester leur intérêt pour la marketplace locale.

\subsection{Intérêt pour la marketplace locale et extension de la zone de chalandise}

Du point de vue des stratégies de développement commercial, on observe que les entrepreneurs qui souhaitent élargir leur zone de chalandise déclarent plus fréquemment être intéressés par la marketplace locale. En effet, $70,7 \%$ des entrepreneurs souhaitant accroître leur zone d'influence se disent intéressés par la plateforme albigeoise, quand seulement $44,4 \%$ du reste de la population l'est. Ainsi, l'ajout de ce dispositif aux solutions de commercialisation existantes semble, en grande partie, tributaire de la propension des entrepreneurs à vouloir (ou non) conquérir des marchés plus larges et plus distants.

\subsection{Analyse des trois facteurs conjugués}

Partant de ces trois variables et les réunissant sous un même tableau de fréquences, nous sommes en mesure de mieux déceler leurs effets conjugués (voir tableau 8). L'étude combinée de ces trois facteurs laisse apparaître un type d'entrepreneurs plus largement intéressés par la marketplace locale : il s'agit des responsables d'entreprises valorisant les ressources locales, favorables à l'avènement de circuits courts et désirant agrandir leur périmètre d'action commerciale $(84,3 \%$ d'entre eux se déclarent intéressés). Ceux-ci ont tout à gagner de la mise en 
Variations autour de l'adoption de plateformes de vente en ligne : la réception d'un nouveau dispositif marchand par les entrepreneurs albigeois

place d'un tel projet, car ils n'ont rien à craindre de l'instauration d'un processus de sélection locale (qui tournerait clairement à leur avantage), car ils souhaitent s'inscrire dans une démarche de circuit court (facilitée par le gain logistique dérivé de l'adhésion à la plateforme), car, enfin, partant d'une zone de chalandise relativement restreinte (clientèle provenant pour l'essentiel de l'agglomération), ils ambitionnent de capter une clientèle plus large dans le cadre de circuits courts d'échelle départementale, voire régionale.

Tableau 8. Intérêt déclaré pour une marketplace locale en fonction des trois variables identifiées ${ }^{17}$

\begin{tabular}{|c|c|c|c|c|}
\hline $\begin{array}{l}\text { Produit et/ou } \\
\text { assemblé } \\
\text { localement }\end{array}$ & $\begin{array}{l}\text { Volonté } \\
\text { d'adhérer à } \\
\text { un circuit } \\
\text { court }\end{array}$ & $\begin{array}{l}\text { Volonté } \\
\text { d'élargir sa } \\
\text { zone de } \\
\text { chalandise }\end{array}$ & $\begin{array}{l}\text { Intérêt pour la } \\
\text { marketplace } \\
\text { locale }\end{array}$ & $\begin{array}{l}\text { Pourcentage } \\
\text { observé }\end{array}$ \\
\hline \multirow[t]{8}{*}{ Oui } & \multirow[t]{4}{*}{ Oui } & \multirow[t]{2}{*}{ Oui } & Oui & $84,3 \%$ \\
\hline & & & Non & $15,7 \%$ \\
\hline & & \multirow[t]{2}{*}{ Non } & Oui & $63,2 \%$ \\
\hline & & & Non & $36,8 \%$ \\
\hline & \multirow[t]{4}{*}{ Non } & \multirow[t]{2}{*}{ Oui } & Oui & $62,5 \%$ \\
\hline & & & Non & $37,5 \%$ \\
\hline & & \multirow[t]{2}{*}{ Non } & Oui & $12,5 \%$ \\
\hline & & & Non & $87,5 \%$ \\
\hline \multirow[t]{8}{*}{ Non } & \multirow[t]{4}{*}{ Oui } & \multirow[t]{2}{*}{ Oui } & Oui & $63,0 \%$ \\
\hline & & & Non & $37,0 \%$ \\
\hline & & \multirow[t]{2}{*}{ Non } & Oui & $52,9 \%$ \\
\hline & & & Non & $47,1 \%$ \\
\hline & \multirow[t]{4}{*}{ Non } & \multirow[t]{2}{*}{ Oui } & Oui & $37,5 \%$ \\
\hline & & & Non & $62,5 \%$ \\
\hline & & \multirow[t]{2}{*}{ Non } & Oui & $12,5 \%$ \\
\hline & & & Non & $87,5 \%$ \\
\hline
\end{tabular}

\section{Conclusion}

L'adoption des plateformes de vente en ligne, pour être bien comprise, doit tout d'abord être mise en perspective avec les moyens techniques et humains dont disposent les entrepreneurs. Dans la première partie de l'article, nous avons constaté que l'équipement préalable des entreprises (site Internet, annuaires professionnels, réseaux sociaux, blogue, forum) conditionnait en partie l'adoption des marketplaces multisites. Nous avons notamment montré que les associations les plus courantes entre dispositifs e-commerce s'élaboraient autour

${ }^{17} x^{2}=26,823 ;$ ddl $=3 ;$ Sig. $=0,000$. 
de trois principaux outils : un site Internet dédié, le référencement sur un annuaire professionnel, l'inscription sur un réseau social. Ces configurations resserrées autour de la triade site-annuaireréseau social sont donc majoritaires et relèguent l'outil marketplace (et d'autres dispositifs avec lui) au second rang des moyens commerciaux mis en œuvre sur la toile. À ce premier constat s'ajoute l'observation d'un " effet taille » sur l'adoption (ou non) des différents dispositifs e-commerce. En effet, alors que les entreprises de 10 salariés ou plus sont nombreuses à mobiliser simultanément plusieurs dispositifs numériques de vente $(72,7 \%$ d'entre elles disposent de 3 à 6 dispositifs), les plus petites - qui ne bénéficient pas des mêmes ressources doivent quant à elles concentrer leurs efforts sur un nombre restreint de dispositifs faciles d'accès et d'usage $(56,3 \%$ des entreprises de 0 à 1 salarié utilisent 1 à 2 dispositifs). II faut également souligner que l'adoption des marketplaces multi-sites est paradoxalement plus fréquente chez les très petites entreprises $(35,9 \%$ des entreprises de 0 à 1 salarié) qu'elle ne l'est chez les entreprises de 2 à 9 salariés et de 10 salariés ou plus (dont les taux d'adoption sont respectivement de $17,9 \%$ et de $29,3 \%$ ). La marketplace représente, pour ces très petites entreprises, un espace de vente tout à fait central (parfois même unique). Ainsi, les plus petites entreprises sont celles qui adoptent le plus fréquemment l'outil marketplace alors même qu'elles sont proportionnellement plus nombreuses à n'utiliser qu'un ou deux dispositifs : le choix d'adhérer aux marketplaces multi-sites pèse donc très fortement sur leur politique commerciale. À l'inverse, les entreprises plus grandes jouent davantage la carte de la multiplication des techniques et des canaux de vente et ne surinvestissent pas outre mesure ce nouvel outil.

Une fois ces premiers éléments réunis, nous avons cherché à identifier les principaux facteurs présidant d'une part à l'adoption effective des marketplaces multi-sites et, d'autre part, à la déclaration d'intérêt pour une marketplace locale. Le seul invariant repéré entre ces deux formes d'adhésion a trait à la volonté communément partagée - par les entrepreneurs adoptants de marketplaces multi-sites et par ceux intéressés par une marketplace locale - d'élargir leur zone de prospection et de chalandise. Si cet élément pèse dans les deux cas considérés, c'est que recourir à une plateforme offre non seulement une chance d'atteindre des publics lointains (géographiquement 
Variations autour de l'adoption de plateformes de vente en ligne : la réception d'un nouveau dispositif marchand par les entrepreneurs albigeois

distants), mais également de toucher des publics jusqu'alors tenus à distance malgré leur proximité spatiale (car placés hors des circuits informationnel et promotionnel couramment employés). Concernant l'adoption des marketplaces multi-sites, il ne fait aucun doute que l'interface numérique permet de mettre en relation, à grande échelle, un large contingent d'acteurs économiques qui font très peu de cas de la distance physique qui les sépare (multitude éclatée). Pour ce qui est de la déclaration d'intérêt en faveur d'une marketplace locale, on observe également que l'extension du périmètre commercial compte pour beaucoup dans la volonté affichée par les entrepreneurs. Ceci n'a cependant rien d'antinomique avec l'ambition concomitamment portée de favoriser l'avènement de circuits courts, car une part non négligeable des entreprises favorables à une telle relocalisation des échanges commerciaux capte aujourd'hui une clientèle pour l'essentiel composée d'individus habitant l'agglomération et envisagent, par conséquent, d'étendre leur périmètre d'intervention au département du Tarn ou à ses départements limitrophes. Ainsi, embrasser un territoire plus large concerne tout à la fois les producteurs locaux et les revendeurs, les entrepreneurs disposés à s'inscrire dans une démarche de circuit court et ceux désirant s'ouvrir à une concurrence plus globale.

Après avoir mis au jour cette similarité, il convient maintenant de souligner ce qui sépare les adoptants des marketplaces globales des adoptants en puissance d'une marketplace locale. Sur ce chapitre, on voit bien que la nature locale du projet albigeois a préférentiellement amené à lui des catégories d'entrepreneurs spécifiques. On ne sera pas surpris d'y voir davantage représentées les entreprises valorisant les ressources locales et soucieuses de s'inscrire dans une logique de circuit court. Ces entreprises pensent la plateforme locale à venir comme une opportunité nouvelle d'identifier et d'enrôler un public étant tout à la fois si près (habitant le même espace) et si loin (hors d'atteinte par les moyens classiques de publicisation de l'offre). La marketplace locale représente donc pour eux un dispositif susceptible de conjurer une situation commerciale regrettable : celle du « so close, so far » (Dubuisson et Mallard, 2004).

Cela dit, au-delà du fait que les deux plateformes, de par leur nature différente, intéressent et enrôlent des catégories 
d'adoptants distinctes, il nous faut rappeler, ici, que nous comparons deux régimes d'adoption (ou de pré-adoption) qui ne relèvent absolument pas du même stade d'évolution. Nous avons ainsi constaté qu'à la différence de la déclaration d'intérêt pour une marketplace locale, l'adoption effective des marketplaces multi-sites était davantage tributaire d'impératifs opérationnels, de considérations pragmatiques (existence ou non de personnes-ressources au sein de l'entreprise) et de relais inter-organisationnels (relation au tissu économique environnant, consultation des entreprises partenaires). Ainsi, on retrouve, parmi les trois facteurs les plus discriminants de l'adoption des marketplaces multi-sites, des variables qui sont tout à la fois de nature structurelle (franchisés vs indépendants), organisationnelle (délimitation de la zone de chalandise) et individuelle (capital scolaire de l'entrepreneur), comme autant de mises à l'épreuve variées d'un projet qui, pour se concrétiser, doit bénéficier d'appuis de tous ordres. A contrario, les variables significativement corrélées à la déclaration d'intérêt pour une marketplace locale se limitent au seul volet organisationnelstratégique (extension de la zone de chalandise, coloration locale de la production, intérêt pour les circuits courts). Cela signifie donc que, dans ce premier temps de réflexion, les entrepreneurs se contentent d'un examen hors sol de l'occasion présentée. Seules les perspectives (figurées) de développement de l'entreprise grâce à l'adoption du dispositif prévalent et s'imposent de prime abord au responsable.

Tout processus de réagencement sociotechnique des stratégies commerciales d'une entreprise respecte donc une construction en deux temps. Le premier temps relève d'un absolu probabiliste (quelles sont les chances d'atteindre par cette adoption un meilleur développement de l'activité ?) fondé sur le désir de révéler, par la médiation sociotechnique, la singularité de son offre. Ce premier temps fait assez peu cas des moyens humains nécessaires à la mise en application du nouvel outil de vente ou même des rigidités structurelles conditionnant son adoption. Un second temps est quant à lui traversé par une forme de réalisme "relationniste " (Cochoy, 2012) qui éprouve le projet d'adoption à l'aune de son intégration dans différents collectifs à la fois techniques (la marketplace dans le paysage des solutions existantes), économiques (la concurrence se jouet-elle sur ces nouveaux espaces numériques marchands?) et sociaux (redistribution des rôles au sein de l'entreprise ou de la 
Variations autour de l'adoption de plateformes de vente en ligne : la réception d'un nouveau dispositif marchand par les entrepreneurs albigeois

franchise). Ce second temps fait, au contraire, grand cas de l'important « travail de contextualisation » à engager, c'est-à-dire des dispositions à prendre en interne comme en externe pour faciliter l'appropriation du nouveau dispositif.

\section{Références}

Amabile, S. et Gadille M. (2003). Les NTIC dans les PME: stratégies, capacités organisationnelles et avantages concurrentiels. Revue française de gestion, (144), 43-63.

Arthur, B. A., (1989). Competing technologies, increasing returns, and lock-in by historical events. The Economic Journal, (99), 116-131.

Aoyama, Y. (2003). Sociospatial dimensions of technology adoption recent M-commerce and E-commerce developments. Environment and Planning A, (35), 1201-1221.

Beauvisage, T., Beuscart, J. S., Cardon, V., Mellet, K. et Trespeuch, M. (2013). Notes et avis des consommateurs sur le web: les marchés à l'épreuve de l'évaluation profane. Réseaux, (177), 131-161.

Benavent, C. (2016). Plateformes. Sites collaboratifs, marketplaces, réseaux sociaux... Comment ils influencent nos choix. Limoges, France : FYP éditions.

Beuscart, J. S. et Flichy, P. (2018). Plateformes numériques. Réseaux, (212), 9-22.

Beuscart, J. S., Dagiral, E. et Parasie, E. (2016). Sociologie d'internet. Paris, France : Armand Colin.

Boullier, D. (2016). Sociologie du numérique. Paris, France: Armand Colin.

Brousseau, E. (2003). E-Commerce in France: Did Early Adoption Prevent Its Development?. The Information Society, (19), 45-57.

Brousseau, E. et Chaves, B. (2004). Diffusion and Impact of ECommerce: The French Specific Path. Report for the Center for Research on Information Technology and Organizations, University of California at Irvine, and the U.S. National Science Foundation, Globalization and E-Commerce Project. Repéré 
à http://citeseerx.ist.psu.edu/viewdoc/download?doi=10.1.1.8 $1.7034 \&$ rep $=$ rep $1 \&$ type $=$ pdf

Bullich, V. et Schmitt, L. (2019). Les industries culturelles à la conquête des plateformes ?. tic\&société, 13(1-2), 1-12.

Caillaud, B. et Jullien, B. (2003). Chicken and Egg: Competition among Intermediation Service Providers. Rand Journal of Economics, (34), 521-552

Callon, M. (2017). L'emprise des marchés. Comprendre leur fonctionnement pour pouvoir changer les choses. Paris, France : La Découverte.

Calvignac, C. (2012a). L'agent économique dormant. Enquête sur la capitalisation professionnelle de signalements communautaires. Sociologie du travail, 54(2), 217-232.

Calvignac, C. (2012b). La fibre communautaire du lien marchand. Dans F. Cochoy (dir.), Du lien marchand. Comment le marché fait société. Toulouse, France: Presses universitaires du Midi.

Cardon, D. et Casilli, A. (2015). Qu'est-ce que le Digital Labor ?. Bry-sur-Marne, France : Institut national de l'audiovisuel.

Cochoy, F. (2003). La « toile " comme force des points faibles ? Vers de petites entreprises «internetionales". Réseaux, (121), 119-144.

Cochoy, F. (2004). La captation des publics. C'est pour mieux te séduire, mon client.... Toulouse, France: Presses universitaires du Midi.

Cochoy, F. (2012). Du lien marchand. Comment le marché fait société. Toulouse, France : Presses universitaires du Midi.

Dubuisson-Quellier, S. (2003). Contacts et relations au marché chez les très petites entreprises. Réseaux, (21), 19-42.

Dubuisson, S. et Mallard, A. (2004, août). So close, so far: How very small businesses use information and communication technologies to interact with their local markets. Communication présenté lors de la 4S-EASST Conference, Paris.

Evans, D. S. et Schmalensee, R. (2016). Matchmakers: The New Economics of Multisided Platforms. Boston, MA : Harvard Business Review Press. 
Variations autour de l'adoption de plateformes de vente en ligne : la réception d'un nouveau dispositif marchand par les entrepreneurs albigeois

FEVAD. (2017). Les chiffres clés : E-commerce aux particuliers. Repéré à https://www.fevad.com/chiffres-cles-cartographie-ecommerce-2017/

FEVAD. (2018). Les chiffres clés : E-commerce aux particuliers. Repéré à https://www.fevad.com/chiffres-cles-cartographie-ecommerce-2018/

Flichy, P. (2010). Le sacre de l'amateur. Sociologie des passions ordinaires à l'ère numérique. Paris, France : Le Seuil.

Flichy, P. (2017). Les nouvelles frontières du travail à l'ère numérique. Paris, France : Le Seuil.

Gadille, M. et d'Iribarne, A. (2000). La diffusion d'Internet dans les PME: motifs d'adoption, réseaux et ressources mobilisées. Réseaux, (104), 59-92.

Gillespie, T. (2010). The Politics of "Platforms". New Media \& Society, 12(3), 347-364.

Insee. (2014). Le commerce électronique en 2012. Les pureplayers dominent les ventes web du commerce de détail. Insee Première, (1489).

Hagberg, J., Sundstrom, M. et Egels-Zandén, N. (2016). The digitalization of retailing: an exploratory framework. International Journal of Retail \& Distribution Management, 44(7), 694-712.

Hong, W. et Zhu, K. (2005). Migrating to internet-based ecommerce: Factors affecting e-commerce adoption and migration at the firm level. Information \& Management, (43), 204-221.

Jullien, N. et Trémembert, J. (2007). Les TIC dans les TPE : un investissement sous contraintes économiques et personnelles. Cahier de recherche de M@rsouin. Repéré à https://hal.archives-ouvertes.fr/hal-02163901/document

Leadbeater, C. et Miller, P. (2004). The Pro-Am Revolution: How Enthusiasts are Changing Our Society and Economy. Londres, Royaume-Uni : Demos.

Lin, H. F. et Lin, S. M. (2008). Determinants of e-business diffusion: A test of the technology diffusion perspective. Technovation, (28), 135-145. 
Luca, M. (2017). Designing online marketplaces: Trust and reputation mechanisms. Innovation Policy and the Economy, 17(1), 77-93.

Mallard, A. (2011). Petit dans le marché : une sociologie de la très petite entreprise. Paris, France : Presses des Mines.

Maxwell, W. et Pénard T. (2016). Quelle régulation pour les plateformes numériques en Europe?. Annales des Mines. Réalités industrielles, (3), 42-46.

Méadel, C. (2006). Le marché eBay : qualification croisée des biens et des personnes. Hermès, La Revue, (44), 79-84.

Oliveira, T. et Martins, M. F. (2010a). Understanding e-business adoption across industries in European countries. Industrial Management \& Data Systems, 110(9), 1337-1354.

Oliveira, T. et Martins, M. F. (2010b). Firms patterns of ebusiness adoption: evidence for the European Union-27. The Electronic Journal Information Systems Evaluation, (13), 4756.

Parker, G., Van Alstyne, M. W. et Choudary, S. P. (2016). Platform Revolution: How Networked Markets Are Transforming the Economy - And How to Make Them Work for You. New York, NY : W.W. Norton and Company.

Plantin, J.-C., Lagoze, C., Edwards, P. N. et Sandvig, C. (2016). Infrastructure studies meet platform studies in the age of Google and Facebook. New Media \& Society, 1-18. https://doi.org/10.1177/1461444816661553

Rallet, A. (2001). Commerce électronique ou électronisation du commerce ?. Réseaux, (106), 17-72.

Rochet, J.-C. et Tirole, J. (2003). Platform competition in twosided markets. Journal of the European Economic Association, 1(4), 990-1029.

Schmalensee, R. et Evans D. S. (2007). Industrial Organization of Markets with Two-Sided Platforms. Competition Policy International, 3(1).

Srnicek, N. (2017). Platform capitalism. Cambridge, RoyaumeUni : John Wiley \& Sons.

Star S. L. et Griesemer J. (1989). Institutionnal ecology, "Translations", and Boundary objects: Amateurs and 
Variations autour de l'adoption de plateformes de vente en ligne : la réception d'un nouveau dispositif marchand par les entrepreneurs albigeois

professionals on Berkeley's museum of vertrebate zoologie. Social Studies of Science, 19(3), 387-420.

Van Heems, R. (2012). Multicanalisation des enseignes. Comment internet transforme les comportements en magasin. Revue française de gestion, (227), 13-29.

Windrum, P. et de Berranger, P. (2002). The adoption of ebusiness technology by SMEs. UNU-MERIT Research Memoranda, (23). Repéré à https://collections.unu.edu/view/ UNU:1124\#viewMetadata

Zhu, K., Kraemer, K. et Xu, S. (2003). Electronic business adoption by European firms: A cross-country assessment of the facilitators and inhibitors. European Journal of Information Systems, (12), 251-268. 